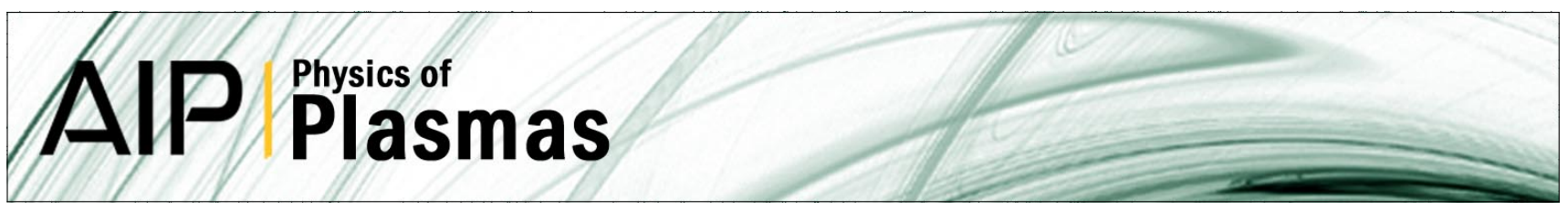

\title{
Low-frequency linear-mode regimes in the tokamak scrape-off layer
}

Annamaria Mosetto, Federico D. Halpern, Sébastien Jolliet, and Paolo Ricci

Citation: Phys. Plasmas 19, 112103 (2012); doi: 10.1063/1.4758809

View online: http://dx.doi.org/10.1063/1.4758809

View Table of Contents: http://pop.aip.org/resource/1/PHPAEN/v19/i11

Published by the American Institute of Physics.

\section{Related Articles}

Spherical torus equilibria reconstructed by a two-fluid, low-collisionality model

Phys. Plasmas 19, 102512 (2012)

Oblique electron-cyclotron-emission radial and phase detector of rotating magnetic islands applied to alignment and modulation of electron-cyclotron-current-drive for neoclassical tearing mode stabilization

Rev. Sci. Instrum. 83, 103507 (2012)

Toroidal rotation of multiple species of ions in tokamak plasma driven by lower-hybrid-waves

Phys. Plasmas 19, 102505 (2012)

Perpendicular dynamics of runaway electrons in tokamak plasmas

Phys. Plasmas 19, 102504 (2012)

Electron cyclotron current drive modelling with parallel momentum correction for tokamaks and stellarators Phys. Plasmas 19, 102501 (2012)

\section{Additional information on Phys. Plasmas}

Journal Homepage: http://pop.aip.org/

Journal Information: http://pop.aip.org/about/about_the_journal

Top downloads: http://pop.aip.org/features/most_downloaded

Information for Authors: http://pop.aip.org/authors

\section{ADVERTISEMENT}

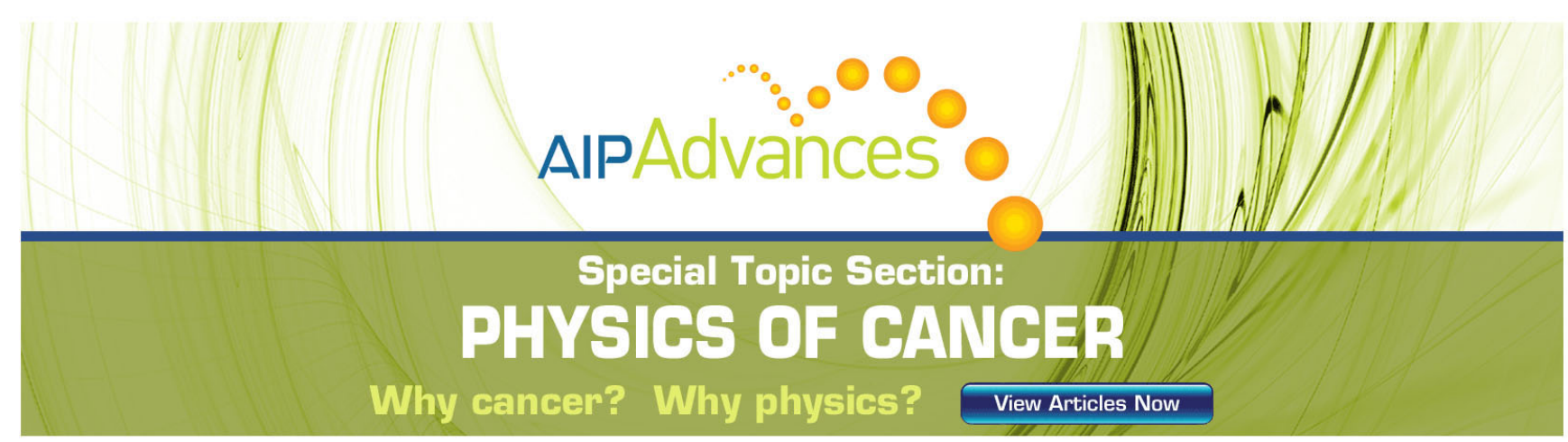




\title{
Low-frequency linear-mode regimes in the tokamak scrape-off layer
}

\author{
Annamaria Mosetto, ${ }^{\text {a) }}$ Federico D. Halpern, Sébastien Jolliet, and Paolo Ricci \\ École Polytechnique Fédérale de Lausanne (EPFL), Centre de Recherches en Physique des Plasmas, \\ Association Euratom-Confédération Suisse, CH-1015 Lausanne, Switzerland
}

(Received 25 July 2012; accepted 24 September 2012; published online 5 November 2012)

\begin{abstract}
Motivated by the wide range of physical parameters characterizing the scrape-off layer (SOL) of existing tokamaks, the regimes of low-frequency linear instabilities in the SOL are identified by numerical and analytical calculations based on the linear, drift-reduced Braginskii equations, with cold ions. The focus is put on ballooning modes and drift wave instabilities, i.e., their resistive, inertial, and ideal branches. A systematic study of each instability is performed, and the parameter space region where they dominate is identified. It is found that the drift waves dominate at high $R / L_{n}$, while the ballooning modes at low $R / L_{n}$; the relative influence of resistive and inertial effects is discussed. Electromagnetic effects suppress the drift waves and, when the threshold for ideal stability is overcome, the ideal ballooning mode develops. Our analysis is a first stage tool for the understanding of turbulence in the tokamak SOL, necessary to interpret the results of non-linear simulations. [http://dx.doi.org/10.1063/ $1.4758809]$
\end{abstract}

\section{INTRODUCTION}

Heat and particle transport in the edge and scrape-off layer (SOL) regions of tokamak plays a fundamental role in determining the overall confinement of a fusion device. ${ }^{1,2}$ It is believed that the transport level observed in these regions results from the low-frequency turbulent plasma dynamics. Since turbulence develops due to the presence of free energy sources, which lead to a number of linearly unstable modes that saturate due to non-linear effects, it is crucial to have a deep insight of the linear properties of the instabilities that develop in these regions in order to understand the observed transport level.

In particular, ballooning modes (BM) and drift waves (DW) are thought to be the instabilities that play the major role in the edge and SOL dynamics. The linear and nonlinear properties of BM and DW have been studied extensively (see, for example, Refs. 3-23). Ballooning modes are driven unstable in the bad curvature region, ${ }^{7-12}$ in the presence of resistivity or finite electron mass, or, in their absence, if the plasma $\beta$ is sufficiently high. Drift waves, on the other hand, arise from $\mathbf{E} \times \mathbf{B}$ convection of the electron density profile, and they become destabilized in the presence of a non-adiabatic electron response, due to, e.g., resistivity or finite electron mass. ${ }^{19-23}$ As a matter of fact, in agreement with experimental results, low-frequency non-linear electromagnetic models (both fluid and gyrofluid) have identified the edge turbulent regimes, ${ }^{3-6,24}$ showing that DW and BM instabilities determine the plasma turbulent dynamics. The relative importance of each mode, however, is still unclear, and non-linear simulations of edge and SOL turbulent dynamics have addressed both instabilities. The SOL region, in particular,

\footnotetext{
a)annamaria.mosetto@epfl.ch.
}

is characterized by a wide range of density gradients and resistivities, ${ }^{24-32}$ allowing the interplay between $\mathbf{E} \times \mathbf{B}$ convection and curvature effects to change considerably, depending on the plasma scenario.

The goal of the present paper is to clarify the relative importance of DW and BM, and of their branches, by defining the linear-mode regimes in the SOL parameter space, i.e., pointing out the fastest growing linear instability given the parameters that characterize a SOL scenario. Our study provides a simple way of identifying the underlying instabilities for a given set of parameters, and it is a starting point for the interpretation of non-linear simulations.

Our stability study is based on a linearization of the drift-reduced Braginskii fluid equations ${ }^{33}$ in $s-\alpha$ geometry with cold ions, and a toroidal limiter placed on the tokamak high-field side. The relative simplicity of the model chosen allows to capture the fundamental properties of both BM and DW by retaining density and temperature gradients, magnetic field curvature, magnetic shear, resistivity, electron inertia, and finite $\beta$ effects. Within this linear fluid framework, we remark that the main parameters characterizing the SOL are: the typical gradient scale length, $L_{n}$, the ratio between the density and the temperature gradient length, $\eta=L_{n} / L_{T}$, the plasma $\beta$, the parallel resistivity, $\nu$, the magnetic shear, $\hat{s}$, the tokamak major and minor radii, $R$ and $a$, and the safety factor $q$.

It is noted that modes other than BM and DW could become unstable in the edge and SOL regions of tokamak plasmas. Among those, we mention peeling-ballooning modes, external kinks, and sheath modes. ${ }^{34-36}$ We remark that in the cold-ion regime considered here, ion temperature gradient modes ${ }^{6,14}$ are excluded, while trapped electron modes are also stable in the SOL due to the fact that the bounce frequency of trapped electrons is smaller than the 
collision frequency. The description of these modes goes beyond the purpose of the present study.

The paper is organized as follows: in Sec. II, we introduce the model that is at the basis of our study. In Secs. III and IV, we present the main characteristics of BM and DW, respectively. Section $\mathrm{V}$ is focused on the transition among the different instabilities, in order to define the linear-mode regimes in the SOL parameter space, while Sec. VI demonstrates how our analysis can be used to interpret the results of SOL studies. Finally, we draw our conclusions in Sec. VII. Appendix A presents a description of the numerical methods used.

\section{THE MODEL}

To study the plasma instabilities present in the SOL, we consider the linearized Braginskii equations (Ref. 33) in the drift-reduced limit, therefore assuming for the perpendicular velocities $\mathbf{V}_{\perp i}=\mathbf{V}_{E \times B}+\mathbf{V}_{* i}+\mathbf{V}_{\text {pol }}$ and $\mathbf{V}_{\perp e}=\mathbf{V}_{E \times B}+\mathbf{V}_{* e}$, where $\mathbf{V}_{E \times B}=(-\nabla \Phi \times \mathbf{B}) / B^{2}$ is the $\mathbf{E} \times \mathbf{B}$ drift velocity, $\mathbf{V}_{* i, e}$ is the ion/electron diamagnetic drift velocity, and $\mathbf{V}_{p o l}$ is the ion polarization velocity (see, e.g., Ref. 37). In the limit $T_{i} \ll T_{e}$, assuming infinite aspect ratio and neglecting the stress tensor, the radially local linearized equations for the perturbed density, $n$, potential, $\Phi$, magnetic vector potential, $\psi=-A_{\|}$, electron temperature, $T_{e}$, and the ion parallel velocity, $V_{\| i}$, are

$$
\begin{aligned}
\frac{\partial n}{\partial t} & =\frac{R}{L_{n}} \frac{\partial \Phi}{\partial y}+\hat{C}\left(T_{e}+n-\Phi\right)+\nabla_{\|} \nabla_{\perp}^{2} \psi-\nabla_{\|} V_{\| i}, \\
\frac{\partial \nabla_{\perp}^{2} \Phi t}{\partial t} & =\hat{C}\left(n+T_{e}\right)+\nabla_{\|} \nabla_{\perp}^{2} \psi, \\
\frac{\partial \psi}{\partial t} \frac{\beta}{2}-\frac{m_{e}}{m_{i}} \frac{\partial}{\partial t} \nabla_{\perp}^{2} \psi & =\nu \nabla_{\perp}^{2} \psi+\nabla_{\|}\left(\Phi-n-1.71 T_{e}\right)+(1+1.71 \eta) \frac{\beta}{2} \frac{R}{L_{n}} \nabla_{\perp}^{2} \psi, \\
\frac{\partial T_{e}}{\partial t} & =\eta \frac{R}{L_{n}} \frac{\partial \Phi}{\partial y}+\frac{2}{3} \hat{C}\left(\frac{7}{2} T_{e}+n-\Phi\right)+\frac{2}{3} 1.71 \nabla_{\|} \nabla_{\perp}^{2} \psi-\frac{2}{3} \nabla_{\|} V_{\| i}, \\
\frac{\partial V_{\| i}}{\partial t} & =-\nabla_{\|}\left(n+T_{e}\right)+\frac{\beta}{2} \frac{R}{L_{n}}(1+\eta) \nabla_{\perp}^{2} \psi .
\end{aligned}
$$

Here, $R$ is the tokamak major radius, $L_{n}$ and $L_{T}$ are the radial scale lenghts of the background density and temperature, $\beta=2 p_{e 0} \mu_{0} / B^{2}$ is the ratio between the equilibrium electron kinetic and the magnetic pressure, $\eta=L_{n} / L_{T}$ is the ratio between the density and the electron temperature gradients length, $\nu=e^{2} n_{0} /\left(m_{i} \sigma_{\|}\right)$is the normalized parallel resistivity, being $\sigma_{\|}=1.96 n_{0} e^{2} \tau_{e} / m_{e}$, the parallel Spitzer conductivity. Background $\mathbf{E} \times \mathbf{B}$ flow is ignored, i.e., the equilibrium $\Phi$ is supposed independent of the radial coordinate. The coordinate $y$ is the poloidal coordinate corresponding, in the infinite aspect ratio approximation, to $y=\theta a$, where $\theta$ is the poloidal angle and $a$ is the minor radius. In particular $0<y<2 \pi a$, with $y=0$ and $y=2 \pi a$ located at the inner mid plane. We normalize $n$ to the equilibrium density $n_{0}, T_{e}$ to the SOL background temperature $T_{e 0}, \Phi$ to $T_{e 0} / e, \psi$ to $c_{s 0} m_{i} \beta / 2 e, V_{\| i}$ to $c_{s 0}=\sqrt{T_{e 0} / m_{i}}$, and the time $t$ to $R / c_{s 0}$. Lengths in the perpendicular direction are normalized to $\rho_{s 0}=c_{s 0} / \Omega_{c i}$ and in the parallel direction to $R$.

According to non-local, linear studies of BM and DW (see Refs. 38 and 39), the scale length in the radial direction is larger than in the poloidal direction, i.e., $k_{y} / k_{r} \sim \sqrt{k_{y} L_{p}} \gg 1$. Therefore, we ignore the radial mode dependence and assume $k_{y} \gg k_{r}$. As a consequence, the curvature operator is defined as

$$
\hat{C}=-2\left[\cos \frac{y}{a}+\hat{s}\left(\frac{y}{a}-\pi\right) \sin \frac{y}{a}\right] \frac{\partial}{\partial y},
$$

where the magnetic shear is $\hat{s}=(a / q) d q / d r, q=a B_{\phi} /\left(R B_{\theta}\right)$ is the safety factor, and the gradients evaluated in the perpendicular direction, lying in the poloidal plane, are

$$
\nabla_{\perp}^{2}=\left[1+\left(\frac{y}{a} \hat{s}-\pi \hat{s}\right)^{2}\right] \frac{\partial^{2}}{\partial y^{2}} .
$$

System (1) is considered in the SOL region, around $r=a$, where the open magnetic field lines end on a toroidal limiter located at the high-field equatorial mid plane $(y=0$ and $y=2 \pi a$ ).

In general, the perturbed quantitites can be written in the form $f_{n}=f_{n}(y) \exp (i n \phi+\gamma t)$, where $n$ is the toroidal mode number, $\phi$ is the toroidal angle, and $\gamma$ is the linear growth rate of the mode. This allows to reduce the system (1) to a onedimensional eigenvalue problem in the $y$ direction for $\gamma$, as the parallel derivative can be evaluated as a combination of the poloidal derivative and the toroidal mode number, as $\nabla_{\|} f_{n}=$ $\left[(a / q) \partial f_{n} / \partial y+i n f_{n}\right] \exp (i n \phi+\gamma t)$. As described in Appendix A, we have developed a toroidal modes decomposition code that solves this eigenvalue problem, whose results are discussed in Sec. VI.

In order to describe the basic properties of the BM and DW (Secs. III and IV), we have also considered the field line following approach to Eqs. (1) in which we assume $\partial / \partial y \rightarrow i k_{y}$, considering a solution in the form $f_{k_{y}}=f_{k_{y}}(z)$ $\exp \left(i k_{y} y+\gamma t\right)$, where $z$ is the parallel coordinate, $0<z<2 \pi q$. Within this approach, the laplacian operator is 


$$
\nabla_{\perp}^{2}=-k_{\perp}^{2}=-k_{y}^{2}\left[1+\left(\frac{z}{q} \hat{s}-\pi \hat{s}\right)^{2}\right]
$$

and the curvature operator is defined as $\hat{C}=i k_{y} C$, where $C=-2[\cos (z / q)+\hat{s}(z / q-\pi) \sin (z / q)]$. More details are given in Appendix A.

In the following two sections, Secs. III and IV, we concentrate our attention on the unstable modes described by the system of Eqs. (1), the BM and DW, using the field line following approach and describing separately their main properties. This is fundamental in order to identify the parameter regime where those modes dominate, which is the subject of Sec. V.

\section{BALLOONING INSTABILITIES}

Ballooning modes are interchange-like modes driven by the curvature of the magnetic field lines and plasma pressure gradient, unstable in the presence of collisions or finite electron mass, or, in their absence, if the plasma $\beta$ is sufficiently high to allow magnetic field lines bending.

For the study of BM, we simplify the system of Eqs. (1), avoiding the coupling with sound waves, i.e., by considering the limit $k_{\|} \ll \gamma$, and therefore neglecting the $V_{\| i}$ dynamics. We also drop the compressibility terms due to magnetic curvature, ascribed to $\mathbf{V}_{E \times B}$ and $\mathbf{V}_{* e}$ convection, in the continuity and temperature equations, because they are much smaller than the $R / L_{n}$ terms. Finally, we neglect the $\nabla_{\|}$terms in the continuity and in temperature equations and the diamagnetic term, $\nabla_{\|}\left(n+1.71 T_{e}\right)$, in Ohm's law, to avoid coupling with DW, therefore assuming $\omega_{*}<\gamma$, where $\omega_{*}=k_{y} R / L_{n}$ is the diamagnetic frequency. In the fluxtube geometry, Eqs. (1) reduce to

$$
\begin{aligned}
\gamma n & =\frac{R}{L_{n}} i k_{y} \Phi, \\
-k_{\perp}^{2} \gamma \Phi & =\hat{C}\left(n+T_{e}\right)-k_{\perp}^{2} \nabla_{\|} \psi \\
\gamma \psi \frac{\beta}{2}+k_{\perp}^{2} \frac{m_{e}}{m_{i}} \gamma \psi & =-k_{\perp}^{2} \nu \psi+\nabla_{\|} \Phi+i k_{\perp}(1+1.71 \eta) \frac{\beta}{2} \frac{R}{L_{n}} \psi \\
\gamma T_{e} & =\eta \frac{R}{L_{n}} i k_{y} \Phi .
\end{aligned}
$$

In the following subsections, we detail the main characteristics of the resistive, the inertial, and the ideal branches of the BM (RBM, InBM, and IdBM, respectively). We find that in all cases, the maximum growth rate is $\gamma_{B}^{\max }=\sqrt{2 R / L_{p}}$. The RBM and InBM have $\gamma \rightarrow \gamma_{B}^{\max }$ for $k_{\|} \rightarrow 0$, therefore the fastest growing mode has the smallest possible $k_{\|}$, approaching the minimum allowed value $k_{\|} \sim 1 / q$. The poloidal mode number $k_{y}$ can vary within a range set by the competition between parallel and perpendicular dynamics (lower $k_{y}$ limit) and by the plasma compressibility (upper $k_{y}$ limit). On the other hand, the IdBM is a global instability that develops with the maximum growth rate at smallest possible $k_{y}$.

\section{A. Resistive ballooning mode}

The resistive branch of the ballooning mode is destabilized by finite parallel resistivity. If electron inertia and electromagnetic effects are neglected, the system of Eqs. (5) can be reduced to the following equation for $\Phi$ :

$$
\hat{\gamma} \Phi\left\{1+[(\hat{z}-\pi) \hat{s}]^{2}\right\}=\sigma_{R} \frac{\partial^{2} \Phi}{\partial \hat{z}^{2}}+\frac{C}{2 \hat{\gamma}} \Phi
$$

where we define $\hat{z}=z / q(0 \leq \hat{z} \leq 2 \pi), \hat{\gamma}=\gamma / \gamma_{B}^{\max }$, and $\sigma_{R}=1 /\left(\gamma_{B}^{\max } k_{y}^{2} q^{2} \nu\right)$, which describes the damping of the mode due to the resistive parallel spread.

Figure 1 shows the growth rate as a function of the magnetic shear $\hat{s}$ and the $\sigma_{R}$ parameter obtained solving the eigenvalue problem of Eq. (6). We observe that the peak of the growth rate is at $\hat{s} \simeq 0.5$ and it decreases asymmetrically moving away from this value. This result agrees with the observations reported in Refs. 6 and 40: for curvature driven modes, positive magnetic shear has a destabilizing effect, while negative shear reduces the region in which the instability can be driven. Moreover, in agreement with our findings, in Ref. 10, it was found that a branch of the resistive ballooning instability was highly unstable up to $\hat{s}=1$. Negative shear stabilization of RBM has been invoked as one of the possible mechanisms behind the formation of transport barriers in the L-H transition (see Ref. 41) as it reduces the fluxes of particles, ${ }^{3,15}$ globally enhancing plasma confinement. The reduction of the growth rate for high values of the $\sigma_{R}$ parameter is due to the competition between the parallel dynamics and the ballooning drive, i.e., the two terms appearing on the right hand side of Eq. (6). The ballooning drive prevails on the parallel dynamics for $k_{\|}^{2} \sigma_{R} \lesssim 1$, leading to an estimate of the value of $k_{y}$ below which the growth rate is reduced by the parallel dynamics, given by $k_{y}^{\text {min }}=$ $1 /\left(2 \pi q \sqrt{\gamma_{B}^{\max } \nu}\right)$ (see Ref. 6).

An analytical estimate for the eigenvalues of Eq. (6) can be calculated in the strong ballooning regime (see, e.g., Refs. 6 and 10). Assuming strong ballooning character of the mode, i.e., a strong localization of the solution near the outer mid plane, we can Taylor expand the curvature operator around that location and derive a Weber-type equation for $\Phi$, of the form

$$
a \frac{d^{2} \Phi}{d z^{2}}+\left(b+c z^{2}\right) \Phi=0,
$$

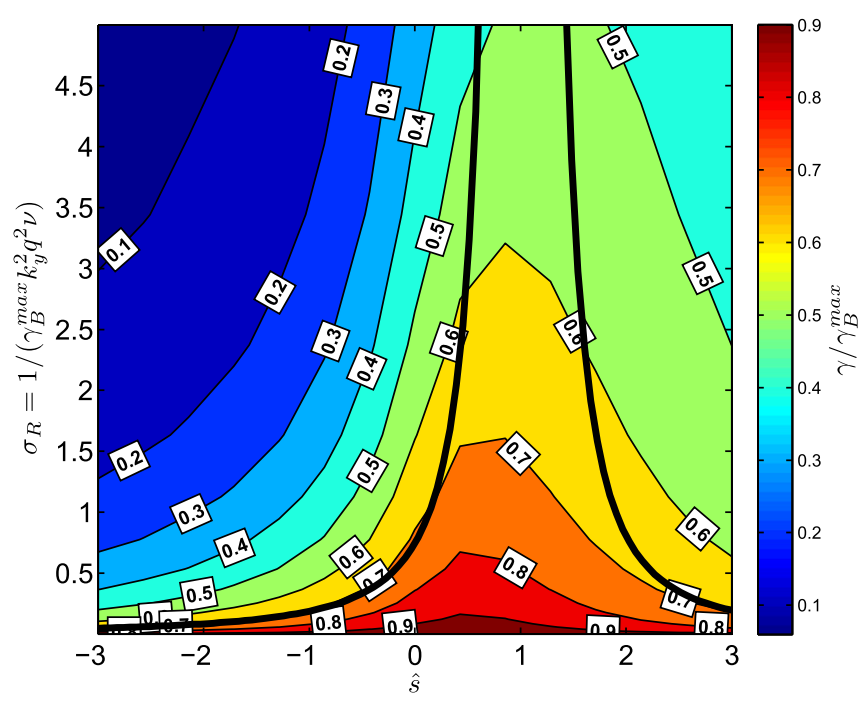

FIG. 1. The normalized growth rate of the resistive ballooning mode, $\gamma / \gamma_{B}^{\max }$, solution of Eq. (6), is plotted as a function of $\hat{s}$ and $\sigma_{R}$; the black line shows the analytical solution given by Eq. (8) for $\gamma / \gamma_{B}^{\max }=0.7$. 
where $a=\sigma_{R} \hat{\gamma}, b=1-\hat{\gamma}^{2}$, and $c=-\hat{\gamma}^{2} \hat{s}^{2}+\hat{s}-1 / 2$. The solution of Eq. (7) is $\Phi=\exp \left(-\lambda^{2} z^{2} / 2\right)$, where $\lambda^{2}=-c / b$, for $\lambda^{2}>0$. Since the coefficients $a, b$, and $c$ have to satisfy $b^{2}+a c=0$, the relation between $\hat{\gamma}, \hat{s}$, and $\sigma_{R}$ is

$$
\sigma_{R}=\frac{2 \hat{\gamma}^{2}-\hat{\gamma}^{4}-1}{\hat{\gamma} \hat{s}-\hat{\gamma} / 2-\hat{\gamma}^{3} \hat{s}^{2}} .
$$

The accuracy of Eq. (8) is higher for localized modes, i.e., with large $\lambda$, which is the case at strong positive and strong negative shear. In Fig. 1, the black line shows the relation between $\sigma_{R}$ and $\hat{s}$ evaluated from Eq. (8) for $\hat{\gamma}=0.7$. Compared to the numerical solution of Eq. (6), one sees that Eq. (8) is able to describe the effect of magnetic shear on the $\operatorname{RBM}$ for $\hat{s} \lesssim 0$ and for $\hat{s} \gtrsim 2$. In fact for $0 \leqq \hat{s} \lesssim 2$, the strong ballooning assumptions are not satisfied and the analytical solution is not accurate. We remark that, according to Eq. (8), the system is unstable even for $\sigma_{R} \rightarrow 0$.

According to the evaluation of the eigenvalues of Eq. (6), $\gamma \rightarrow \gamma_{B}^{\max }$ for $k_{y} \rightarrow \infty$. However, the solution of Eqs. (1) shows that $\gamma \rightarrow 0$ for $k_{y} \rightarrow \infty$. We find that this is due to magnetic curvature induced plasma compressibility, which is not included in the symplified system (5). This effect can be understood by considering a relatively simple model, Eqs. (1) in the $k_{\|}=0$ limit and assuming constant curvature evaluated at the outer mid plane. The linear dispersion relation associated to such a system is ${ }^{18} b_{0}+b_{1} \gamma+b_{2} \gamma^{2}+b_{3} \gamma^{3}=0$, where $b_{0}=20 i k_{y}^{3}\left(2-R / L_{n}\right) / 3, b_{1}=20\left(k_{y}^{2}-1\right) k_{y}^{2} / 3+2(1+\eta) k_{y}^{2}$ $R / L_{n}, b_{2}=20 i k_{y}^{3} / 3$, and $b_{3}=-k_{y}^{2}$. The solution of this dispersion relation shows reduction of the growth rate for $k_{y} \gtrsim 0.3 \gamma_{B}^{\max }$; our numerical tests show that this reduction is due to the compressibility terms in the density and temperature equations. In conclusion, the RBM grows for $k_{y}^{\min }<k_{y}<k_{y}^{\max }$, being $k_{y}^{\min }=1 /\left(2 \pi q \sqrt{\gamma_{B}^{\max } \nu}\right)$ and $k_{y}^{\max }=0.3 \gamma_{B}^{\max }$.

In a previous study (see Ref. 6), BM analysis demonstrated that their growth rate is reduced by diamagnetic effects when $\alpha_{D}=R k_{y}^{\min } /\left(L_{n} \gamma_{B}^{\max }\right)>1$. We observe a reduction of the growth rate at high $k_{y}$ due to compressibility effects, ascribed to both the diamagnetic terms $\left(\hat{C} T_{e}\right.$ and $\left.\hat{C} n\right)$ and the potential term $(\hat{C} \phi)$ in the density and temperature equations. Our approach separates the compressibility damping from the diamagnetic effects in Ohm's law, while in Ref. 6, the two contributions were not clearly separated.

\section{B. Inertial ballooning mode}

In the limit of negligible resistivity and negligible electromagnetic effects, one finds the inertial branch of the BM instability. In this limit, Eqs. (5) can be reduced to the following equation for $\Phi$ :

$$
\hat{\gamma} \Phi\left\{1+[(\hat{z}-\pi) \hat{s}]^{2}\right\}=\sigma_{I n}^{2} \hat{\gamma} \frac{\partial^{2} \Phi}{\partial \hat{z}^{2}}+\frac{C}{2 \hat{\gamma}} \Phi,
$$

where $\sigma_{I n}=\sqrt{m_{i}} /\left(\gamma_{B}^{\max } k_{y} q \sqrt{m_{e}}\right)$, which describes the damping of the mode due to the inertial parallel spread.

In Fig. 2, we show the growth rate as a function of $\hat{s}$ and $\sigma_{\text {In }}$, solution of the eigenvalue problem of Eq. (9). We observe that the reduction of $\gamma$ due to the magnetic shear is asymmetric with respect to the peak value occurring at $\hat{s} \simeq 0.5$. As for the RBM, we remark that the diminution of the growth rate with $\sigma_{I n}$ is due to the competition between the ballooning drive and the parallel dynamics terms appearing on the right hand side of Eq. (9). By comparing the two terms on the right hand side of Eq. (9), we find the minimum value of $k_{y}$, below which we have a considerable suppression of the growth rate, which is $k_{y}^{\min }=\sqrt{m_{i}} /\left(2 \pi q \gamma_{B}^{\max } \sqrt{m_{e}}\right)$.

As in the case of RBM, it is possible to solve Eq. (9) within the strong ballooning limit (see Refs. 6 and 10). In this case, the coefficients of the Weber equation, Eq. (7), are $a=\sigma_{I n}^{2}, \quad b=-\hat{\gamma}^{2}+1$, and $c=-\hat{\gamma}^{2} \hat{s}^{2}+\hat{s}-1 / 2$ and the relation between $\sigma_{I n}, \hat{s}$, and $\hat{\gamma}$ is given by

$$
\sigma_{\text {In }}=\sqrt{\frac{2 \hat{\gamma}^{2}-\hat{\gamma}^{4}-1}{\hat{s}-1 / 2-\hat{\gamma}^{2} \hat{s}^{2}}} .
$$

In Fig. 2, the black line shows the relation between $\sigma_{\text {In }}$ and $\hat{s}$ given by Eq. (10) for $\hat{\gamma}=0.7$ compared to the numerical solution of Eq. (9), as in the RBM case. We notice that the agreement between the analytical and the numerical solution is good for $\hat{s} \lesssim 0$ and for $\hat{s} \gtrsim 2$. In fact for $0 \leqq \hat{s} \lesssim 2$, the strong ballooning assumption is not valid and the analytical solution, Eq. (10), is not accurate. We remark that, according to Eq. (10), the system is unstable even for $\sigma_{I n} \rightarrow 0$. As stated for the RBM case, also for the InBM, the compressibility reduces the growth rate for $k_{y} \gtrsim 0.3 \gamma_{B}^{\max }{ }^{18}$

\section{Ideal ballooning mode}

The ideal ballooning instability persists in the absence of plasma resistivity and electron inertia, and it is characterized by magnetic field lines bending outward in the bad curvature region due to interchange drive. In the limit of negligible resistivity, $\nu$, and negligible electron mass, $m_{e}$, the system of Eqs. (5) can be reduced to the following equation for $\Phi$

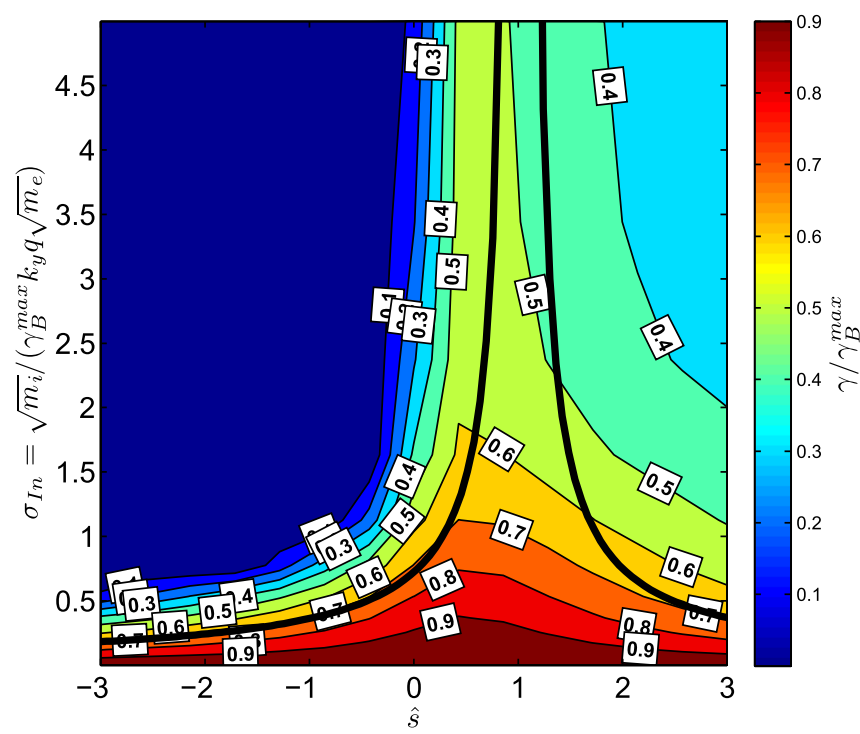

FIG. 2. The normalized growth rate of the inertial ballooning mode, $\gamma / \gamma_{B}^{\max }$ solution of Eq. (9), is plotted as a function of $\hat{s}$ and $\sigma_{I n}$; the black line shows the analytical solution given by Eq. (10) for $\gamma / \gamma_{B}^{\max }=0.7$. 


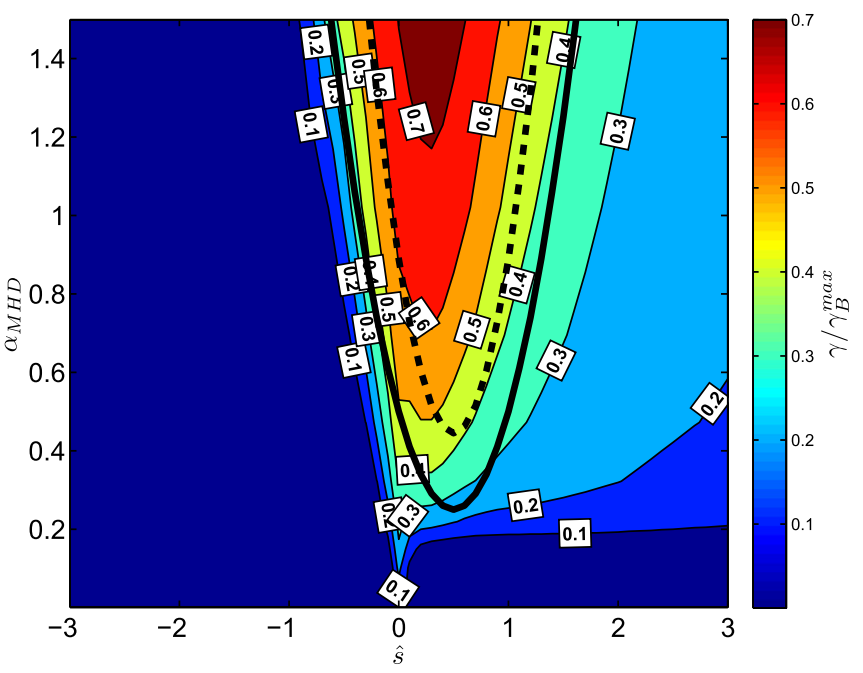

FIG. 3. The normalized growth rate of the ideal ballooning mode, $\gamma / \gamma_{B}^{\max }$, solution of Eq. (11), is plotted as a function of $\hat{s}$ and $\alpha_{M H D}$; the dotted black line shows the analytical solution for $\gamma / \gamma_{B}^{\max }=0.5$, while the continuous black line shows the ideal marginal stability, $\gamma=0$, both given by Eq. (12).

$$
-\hat{\gamma} \Phi\left\{1+[(\hat{z}-\pi) \hat{s}]^{2}\right\}=-\frac{\left\{1+[(\hat{z}-\pi) \hat{s}]^{2}\right\}}{\alpha_{M H D} \hat{\gamma}} \frac{\partial^{2} \Phi}{\partial \hat{z}^{2}}-\frac{C}{2 \hat{\gamma}} \Phi,
$$

where $\alpha_{M H D}=q^{2} \beta(1+\eta) R / L_{n}$. The growth rate as a function of $\hat{s}$ and $\alpha_{M H D}$ is shown in Fig. 3, as a solution of the eigenvalue problem of Eq. (11). When the parallel stabilization is overcome, i.e., for $\alpha_{M H D} \sim 1$, the IdBM is unstable independently of $k_{y}$ (see Ref. 6), since $\alpha_{M H D}$ is independent of $k_{y}$. The magnetic shear has a stabilizing effect that is not symmetric with respect to the peak value occurring at $\hat{s} \simeq 0.5$, the damping of the growth rate for $\hat{s}<0$ being more effective than for $\hat{s}>0$. In the strong ballooning regime, the coefficients of the Weber equation, Eq. (7), associated with Eq. (11) are: $a=1, b=\alpha_{M H D}\left(1-\gamma^{2}\right)$, and $c=\alpha_{M H D}\left(-\hat{s}^{2}+\hat{s}-1 / 2\right)$. For the IdBM case, the analytical solution in the strong ballooning limit leads to the relation among $\alpha_{M H D}, \hat{s}$, and $\hat{\gamma}$ given by

$$
\alpha_{M H D}=\frac{\hat{s}-1 / 2-\hat{s}^{2}}{2 \hat{\gamma}^{2}-\hat{\gamma}^{4}-1} .
$$

The black continuous line in Fig. 3 shows the relation between $\alpha_{M H D}$ and $\hat{s}$, Eq. (12), for $\hat{\gamma}=0$ (marginal ideal stability), while the dotted line shows the same relation for $\hat{\gamma}=0.5$, compared to the numerical solution of Eq. (11). The numerical solution of Eq. (11) shows good agreement with the solid curve in Fig. 1 of Ref. 7, which was obtained following the hypothesis described in Ref. 42. In that case, the marginal ideal stability was computed from the ideal MHD energy principles, imposing zero boundary conditions in the poloidal direction. We remark that, according to Eq. (12), the system is stable for $\alpha_{M H D} \rightarrow 0$, showing the existence of a pressure threshold for the destabilization of the IdBM. As in the RBM and InBM cases, when compressibility effects are retained in Eqs. (1), we verified a reduction of the growth rate with increasing $k_{y}$ that becomes important for $k_{y} \gtrsim 0.3 \gamma_{B}^{\max }{ }^{18}$ Therefore, the maximum growth rate of the IdBM develops for $k_{y} \rightarrow 0$.

\section{DRIFT WAVE INSTABILITY}

The DW instability is caused by $\mathbf{E} \times \mathbf{B}$ convection of the plasma pressure accompanied by the breaking of the electron adiabaticity in Ohm's law, which is due to resistivity or finite electron mass. ${ }^{17,18}$ Electromagnetic effects stabilize the DW instability, as shown in Sec. VE. For DW, typically $\gamma \sim \omega_{*}, k_{y} \sim 1$, while $k_{\|}$takes a finite value. In order to model the DW instability we simplify Eqs. (1) by neglecting the sound waves coupling, i.e., by assuming $\gamma \gg k_{\|}$. Moreover, we turn off the ballooning drive, i.e., the curvature terms in the vorticity equation, in order to exclude $\mathrm{BM}$ from the system. We also neglect the compressibility terms in the continuity and temperature equations, since they have a stabilizing effect that we ignore for sake of simplicity. The reduced system of equations able to take into account the fundamental elements of the DW is
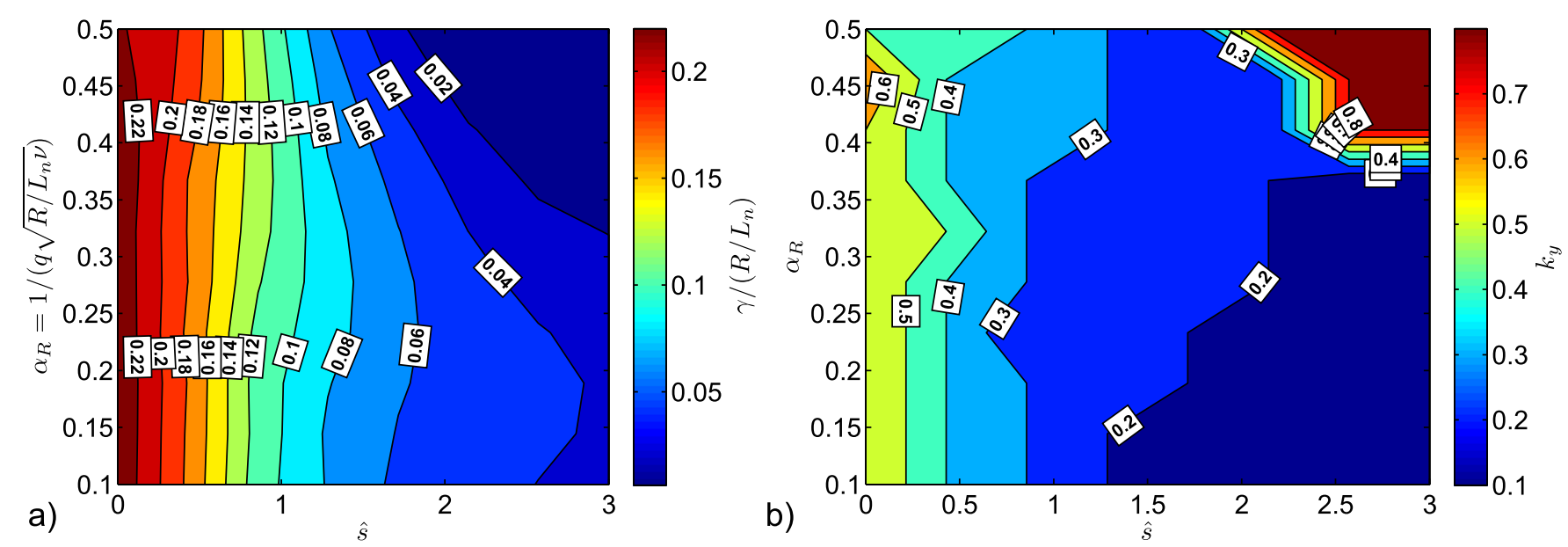

FIG. 4. The normalized growth rate of the resistive drift wave, $\gamma L_{n} / R$, maximized over $k_{y}$, (a) and $k_{y}$ of the maximum growth rate (b), solution of Eq. (14), are plotted as a function of $\hat{s}$ and $\alpha_{R} . \hat{s}>0$ is represented since Eq. (14) is invariant for $\hat{s} \rightarrow-\hat{s}$ transformation. 


$$
\begin{aligned}
\gamma n= & i k_{y} \frac{R}{L_{n}} \Phi-k_{\perp}^{2} \nabla_{\|} \psi,-k_{\perp}^{2} \gamma \Phi=-k_{\perp}^{2} \nabla_{\|} \psi \\
\gamma \psi \frac{\beta}{2}+\frac{m_{e}}{m_{i}} \gamma k_{\perp}^{2} \psi= & -k_{\perp}^{2} \nu \psi+\nabla_{\|}\left(\Phi-n-1.71 T_{e}\right) \\
& +i k_{\perp}(1+1.71 \eta) \frac{\beta}{2} \frac{R}{L_{n}} \psi, \\
\gamma T_{e}= & i k_{y} \eta \frac{R}{L_{n}} \Phi-k_{\perp}^{2} \frac{2}{3} 1.71 \nabla_{\|} \psi .
\end{aligned}
$$

We analyze this system in more detail by separating the resistive and the inertial branches of the DW.

\section{A. Resistive drift waves}

In the case of resistive DW (RDW), the adiabaticity is broken by the presence of a finite parallel resistivity. Neglecting electron inertia and electromagnetic effects, the system of Eqs. (13) is reduced to the following equation for $\Phi$ :

$$
\check{\gamma} k_{\perp}^{2} \Phi=\frac{\partial^{2} \Phi}{\partial \check{z}^{2}}+2.94 \frac{\partial^{2}\left(k_{\perp}^{2} \Phi\right)}{\partial \check{z}^{2}}-\frac{1}{\check{\gamma}}\left[i k_{y}(1+1.71 \eta)\right] \frac{\partial^{2} \Phi}{\partial \check{z}^{2}},
$$

where $\check{z}=z \sqrt{\nu R / L_{n}}, \check{\gamma}=\gamma L_{n} / R, k_{\perp}^{2}=k_{y}^{2}\left[1+\left(\check{z} \alpha_{R} \hat{s}-\pi \hat{s}\right)^{2}\right]$, and $\alpha_{R}=\sqrt{L_{n}} /(q \sqrt{\nu R})$.

In Fig. 4, the growth rate of the fastest growing mode, found from Eq. (14), and the corresponding $k_{y}$ are shown as a function of $\hat{s}$ and $\alpha_{R}$, assuming $\eta=1$. Magnetic shear damps the instability almost independently of $\alpha_{R}$ in the observed range of values, with the maximum growth rate at $\hat{s}=0$. The typical wavenumber of the fastest growing mode is in the range $0.2<k_{y}<0.8$. We remark that for $\hat{s}=0$, with the substitution $\partial / \partial z \rightarrow i k_{\|}$, Eq. (14) can be reduced to an algebraic equation, $\nu k_{y}^{2} \gamma^{2}+k_{\|}^{2}\left(1+2.94 k_{y}^{2}\right) \gamma+(1+$ $1.71 \eta) i k_{\|}^{2} k_{y} R / L_{n}=0$, with a maximum growth rate of $\gamma_{R D W}^{\max } \simeq 0.085 \quad(1+1.71 \eta) R / L_{n} \quad$ at $\quad k_{y} \simeq 0.57 \quad$ and $k_{\|} \simeq 0.24 \sqrt{\nu R / L_{p}}{ }^{18}$

We note that the influence of magnetic shear on the RDW has been discussed, for example, in Ref. 43, in the collisionless limit, and in Ref. 44, with the inclusion of resistiv- ity. For a constant value of $R / L_{n}$, in both cases it has been found that the DW instability in a sheared slab geometry is unconditionally stable. We find that the growth rate of DW is suppressed by shear effects, but the instability is not unconditionally stable for $\hat{s} \neq 0$. In Refs. 43 and 44, the radially non-local DW dispersion relation is studied, neglecting the electron temperature dynamics and assuming $k_{\|}=0$ at the center of the flux tube. In our approach, we allow $k_{\|} \neq 0$, leading to the development of an unstable DW instability, even in the presence of magnetic shear.

\section{B. Inertial drift waves}

In the inertial branch of the DW (InDW), the electron adiabaticity is broken by the presence of a finite electron mass. Neglecting resistivity and electromagnetic effects, the system of Eqs. (13) can be reduced to the following equation for $\Phi$ :

$$
\check{\gamma}^{2} k_{\perp}^{2} \Phi=\frac{\partial^{2} \Phi}{\partial \bar{z}^{2}}+2.94 \frac{\partial^{2}\left(k_{\perp}^{2} \Phi\right)}{\partial \bar{z}^{2}}-\frac{1}{\check{\gamma}}\left[i k_{y}(1+1.71 \eta)\right] \frac{\partial^{2} \Phi}{\partial \bar{z}^{2}},
$$

where $\bar{z}=z R \sqrt{m_{e}} /\left(L_{n} \sqrt{m_{i}}\right), k_{\perp}^{2}=k_{y}^{2}\left[1+\left(\check{z} \alpha_{I} \hat{s}-\pi \hat{s}\right)^{2}\right]$, and $\alpha_{I}=L_{n} \sqrt{m_{i}} /\left(q R \sqrt{m_{e}}\right)$.

In Fig. 5, the solution of Eq. (15) and the $k_{y}$ related to the maximum growth rate are shown as a function of $\hat{s}$ and $\alpha_{I}$, assuming $\eta=1$. As for the RDW, the maximum growth rate is reached for $\hat{s}=0$ and magnetic shear causes a damping of the instability, almost independently of $\alpha_{I}$ in the observed range of values. We note that the magnetic shear damps more efficiently the RDW instability than the InDW instability. For example, the growth rate of the InDW is reduced approximately to $30 \%$ of the shearless value at $\hat{s}= \pm 3$ while, in the RDW case, the growth rate is reduced to approximately $10 \%$. The typical wavenumber of the fastest growing mode is in the range $0.35<k_{y}<0.6$. For the $\hat{s}=0$ case, Eq. (15) can be reduced to an algebraic equation, $m_{e} / m_{i} k_{y}^{2} \gamma^{3}$ $+k_{\|}^{2}\left(1+2.94 k_{y}^{2}\right) \gamma+(1+1.71 \eta) i k_{\|}^{2} k_{y} R / L_{n}=0$, with a maximum growth rate given by $\gamma_{\operatorname{lnD} W}^{\max } \simeq 0.17(1+1.71 \eta) R / L_{n}$, at $k_{y} \simeq 0.57$ and $k_{\|} \simeq 02 R \sqrt{m_{e}} /\left(L_{p} \sqrt{m_{i}}\right) .{ }^{18}$ The maximum growth rate is double the value obtained for RDW.
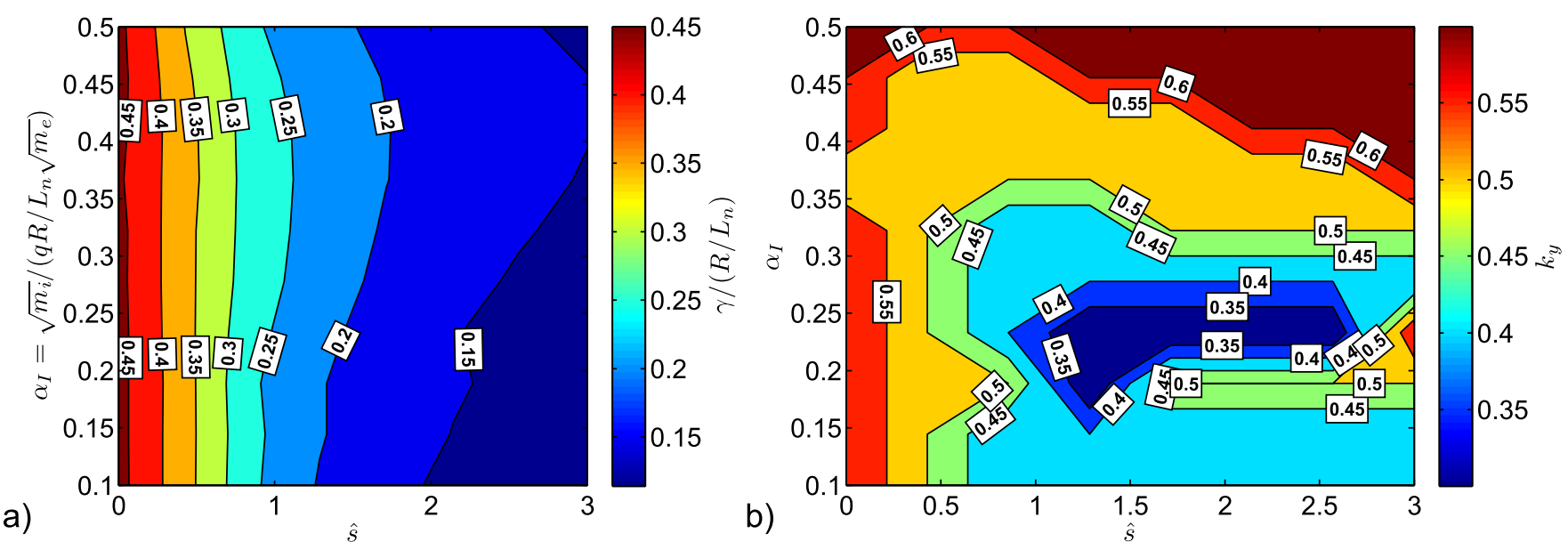

FIG. 5. The normalized growth rate of the inertial drift wave, $\gamma L_{n} / R$, maximized over $k_{y}$, (a) and $k_{y}$ of the maximum growth rate (b), solution of Eq. (15), are plotted as a function of $\hat{s}$ and $\alpha_{I} . \hat{s}>0$ is represented since Eq. (15) is invariant for $\hat{s} \rightarrow-\hat{s}$ transformation. 


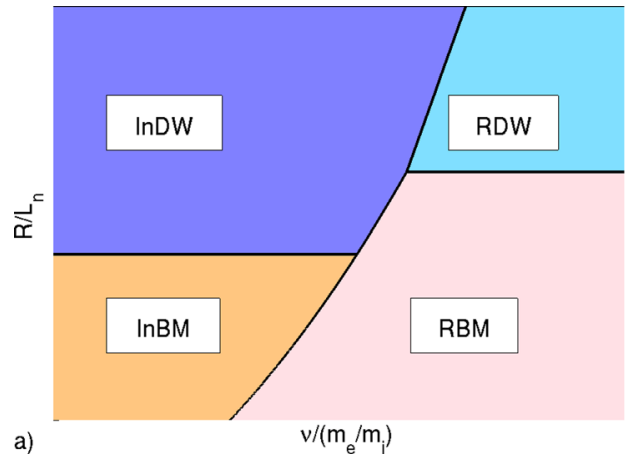

\section{PARAMETER SPACE OF THE LINEAR INSTABILITIES}

We now identify the parameter space of the previously described linear instabilities. Our goal is to provide a framework according to which, given the set of parameters necessary to characterize the SOL, it is possible to state which is the dominant linear mode, i.e., the one that has the fastest growth rate. Within our model, the parameters necessary to characterize the SOL are: $R / L_{n}, \nu, m_{e} / m_{i}, \beta, \hat{s}$, and $q$. In Fig. 6, the different regimes of linear instabilities are schematically identified in the parameter space. Our analysis starts from the electrostatic limit, $\beta=0$, represented in Fig. 6(a). Since DW have a growth rate of the order $\gamma \sim \omega_{*} \sim R / L_{n}$, while BM growth rate scales as $\sqrt{R / L_{n}}$, we expect the DW to overcome the BM growth rate for sufficiently steep density gradients. In fact, four regimes can be distinguished: at high values of $R / L_{n}$, the DW is the dominant instability, the resistive branch prevailing at high resistivity, and the inertial branch at low resistivity. For low values of $R / L_{n}$ BM dominate, in particular, the resistive branch at high resistivity and inertial branch at low resistivity. Finite $\beta$ effects are described in Fig. 6(b). At high values of $R / L_{n}$, for increasing values of $\beta$, first DW suppression due to electromagnetic effects is observed and then the IdBM becomes unstable, once the $\alpha_{M H D}$ threshold is overcome. For small values of $R / L_{n}$, the RBM and the InBM dominate at small $\beta$ and the IdBM at high $\beta$.

In the following paragraph, we first provide a description of the transition among the different instabilities in the electrostatic case. We then discuss the role of electromagnetic effects.

\section{A. Transition between resistive ballooning mode and resistive drift wave}

An estimate of the transition between the RDW and RBM can be obtained by comparing their maximum growth rates. In the shearless case, a very simple estimate can be obtained by equating the maximum growth rate for RDW, $\gamma_{R D W}^{\max }$, defined in Sec. IV A, to the maximum growth rate for RBM, $\gamma_{B}^{\max }$, defined in Sec. III. One obtains a transition value of $R / L_{n}$, which is $R / L_{n}=2(1+\eta) /[0085(1+1.71 \eta)]^{2} \simeq$ 75.2 at $\eta=1$.

In the general case, the threshold value of $R / L_{n}$ depends on $\hat{s}, \sigma_{R}$ and $\alpha_{R}$ and is obtained by comparing the solutions of Eqs. (6) and (14), namely $\gamma_{R B M}$ and $\gamma_{R D W}$, respectively. We identify the $R / L_{n}$ threshold in correspondence to
FIG. 6. Sketch of the linear instability regimes in the parameter space: electrostatic limit (a) and full electromagnetic analysis (b). Different colours identify the parameter space of the different instabilities: resistive ballooning (pink), inertial ballooning (orange), resistive drift wave (light blue), inertial drift wave (dark blue), ideal ballooning (violet), and region of suppression of drift waves (green).

$\gamma_{R D W} / \gamma_{R B M}=1$. In the following analysis, we fix $\alpha_{R}=0.35$, since the DW depend weakly on this parameter. In Fig. 7, we show the $R / L_{n}$ threshold as a function of $\hat{s}$ and $\sigma_{R}$. The $R / L_{n}$ threshold decreases for increasing $\sigma_{R}$, since the RBM is suppressed by the parallel dynamics. For $\hat{s}=0$, while at $\sigma_{R} \simeq 0$, the transition between RDW and RBM occurs at $R / L_{n} \simeq 75$ (in agreement with our analytical estimate), at $\sigma_{R} \simeq 0.5$, the RDW grows faster than the RBM for $R / L_{n} \geq 45$. The $R / L_{n}$ threshold decreases to $R / L_{n} \simeq 15$ for $\hat{s}=0$ at $\sigma_{R} \simeq 3$. The decrease of the $R / L_{n}$ threshold is more noticeable for $\hat{s}<0$, as the RBM is more efficiently suppressed by negative shear (see Fig. 1) and the asymmetry with respect to $\hat{s}=0$ becomes evident at high values of $\sigma_{R}$. In the white region, the $R / L_{n}$ threshold is at values greater than 300 and the RBM always prevails on the RDW.

\section{B. Transition between inertial ballooning mode and inertial drift waves}

In order to estimate the threshold value of $R / L_{n}$ above which the InDW grows faster than the InBM, we can proceed as for the resistive case. For $\hat{s}=0$, a simple analytical estimate of the threshold can be obtained by equating the maximum growth rate for InDW, $\gamma_{\operatorname{InDW}}^{\max }$, defined in

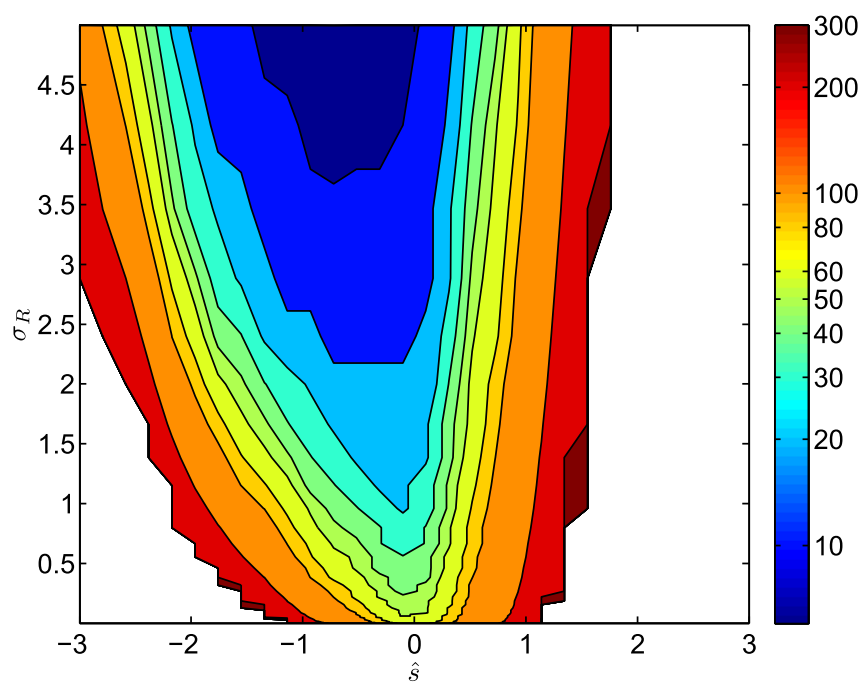

FIG. 7. Transition between resistive drift waves and resistive ballooning mode. The $R / L_{n}$ value for which the growth rate of the RDW, solution of Eq. (14), and of the RBM, solution of Eq. (6), are equal, $\gamma_{R D W}=\gamma_{R B}$, is plotted as a function of $\hat{s}$ and $\sigma_{R}$. In the white region, the RBM always prevails on the RDW for $R / L_{n}>300$. 


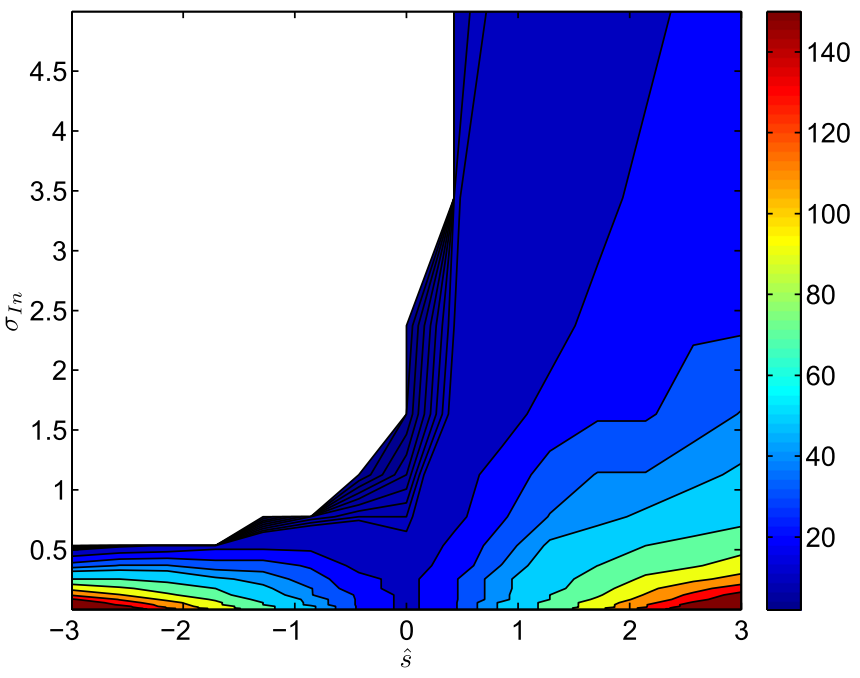

FIG. 8. Transition between inertial drift waves and inertial ballooning mode. The $R / L_{n}$ value for which the growth rate of the InDW, solution of Eq. (15), and of the InBM, solution of Eq. (9), are equal, $\gamma_{I n D W}=\gamma_{I n B}$, is plotted as a function of $\hat{s}$ and $\sigma_{I n}$. In the white region, the InDW always prevail on the InBM.

Sec. IV B, to the maximum growth rate for InBM, $\gamma_{B}^{\max }$, defined in Sec. III. The normalized gradient below which the InBM growth rate is larger than the one for the InDW is $R / L_{n}=2(1+\eta) /[017(1+1.71 \eta)]^{2} \simeq 18.8$ at $\eta=1$. In general, the threshold depends on $\hat{s}, \sigma_{I n}$, and $\alpha_{I}$ and can be evaluated comparing the solution of Eqs. (9) and (15), $\gamma_{\text {InBM }}$ and $\gamma_{\text {InDW }}$, respectively, identifying the $R / L_{n}$ threshold in correspondence to $\gamma_{I n D W} / \gamma_{\operatorname{InBM}}=1$. In Fig. 8 , we show the $R / L_{n}$ threshold as a function of $\hat{s}$ and $\sigma_{I n}$, for $\alpha_{I}=0.30$. The $R / L_{n}$ threshold decreases for increasing $\sigma_{I n}$, since the InBM is suppressed by the parallel dynamics. As for the RBM, the decrease is more evident for $\hat{s}<0$. For $\sigma_{\text {In }} \simeq 0$, we observe that the $R / L_{n}$ threshold is very close to the analytical estimate previously calculated for $\hat{s}=0$ and that, because of the shear damping of the InDW, at $\hat{s}= \pm 1$, the transition occurs at $R / L_{n} \simeq 55$. The threshold decreases to $R / L_{n} \simeq 10$, due to the smaller growth rate of the InBM at $\sigma_{I n} \simeq 0.5$ and $\hat{s}=0$. In the white region of Fig. 8, the InDW always prevails on the InBM.

\section{Transition between resistive drift wave and inertial drift wave}

In the parameter space region where $R / L_{n}$ is sufficiently high, and therefore the DW are the dominant instability, the relative influence of the resistive term with respect to the inertial term governs the transition between the RDW and the InDW. The threshold value of resistivity for the transition between these two branches of the DW can be roughly estimated by balancing the resistive term and the inertial term in Ohm's law: if $\nu>\gamma m_{e} / m_{i}$, resistive effect dominates, leading therefore to the development of the RDW instability, otherwise inertial effects do, i.e., the InDW prevail.

A more precise estimate of the transition value of the resistivity can be obtained by studying the behaviour of the system of Eqs. (13), considering the $\beta=0$ limit, as a function of $\delta=\nu L_{n} m_{i} /\left(R m_{e}\right)$, which defines the ratio between the resistive and inertial effects. In Fig. 9(a), we plot the growth rate of DW as a function of $\delta$, for different values of $\hat{s}$. From low to high values of $\delta$, one observes the transition from the InDW to the RDW region. The maximum RDW growth rate is half the one for the InDW for $\hat{s}=0$. In general, it is always smaller than the one for InDW, even for $\hat{s} \neq 0$. Therefore, one can obtain the value of $\delta$ at which the transistion takes place, by evaluating the value of $\delta$ at which the growth rate is the average of the growth rates for RDW and InDW. We observe that, for increasing $\hat{s}$, the value of $\delta$ at which the transition from InDW to RDW occurs decreases. This is plotted in Fig. 9(b): the $\delta$ threshold passes from $\delta \sim 3.55$ for $\hat{s}=0$ to $\delta \sim 1.12$ for $\hat{s}=5$.

\section{Transition between resistive ballooning mode and inertial ballooning mode}

The threshold between RBM and InBM has been calculated by comparing the growth rate of the two linear modes, solutions of Eqs. (6) and (9), $\gamma_{R B M}$ and $\gamma_{I n B M}$, respectively. In the resistive limit, $\gamma$ is a function of $\hat{s}$ and $\sigma_{R}$ and, in the inertial limit, it depends on $\hat{s}$ and $\sigma_{I n}$, therefore the ratio $\gamma_{I n B M} / \gamma_{R B M}$ has to be evaluated as a function of $\sigma_{R}, \sigma_{I n}$, and $\hat{s}$. We observe that the ratio is larger or smaller than 1 , independently of $\hat{s}$, in a wide region of the plane $\left(\sigma_{R}, \sigma_{I n}\right)$. In
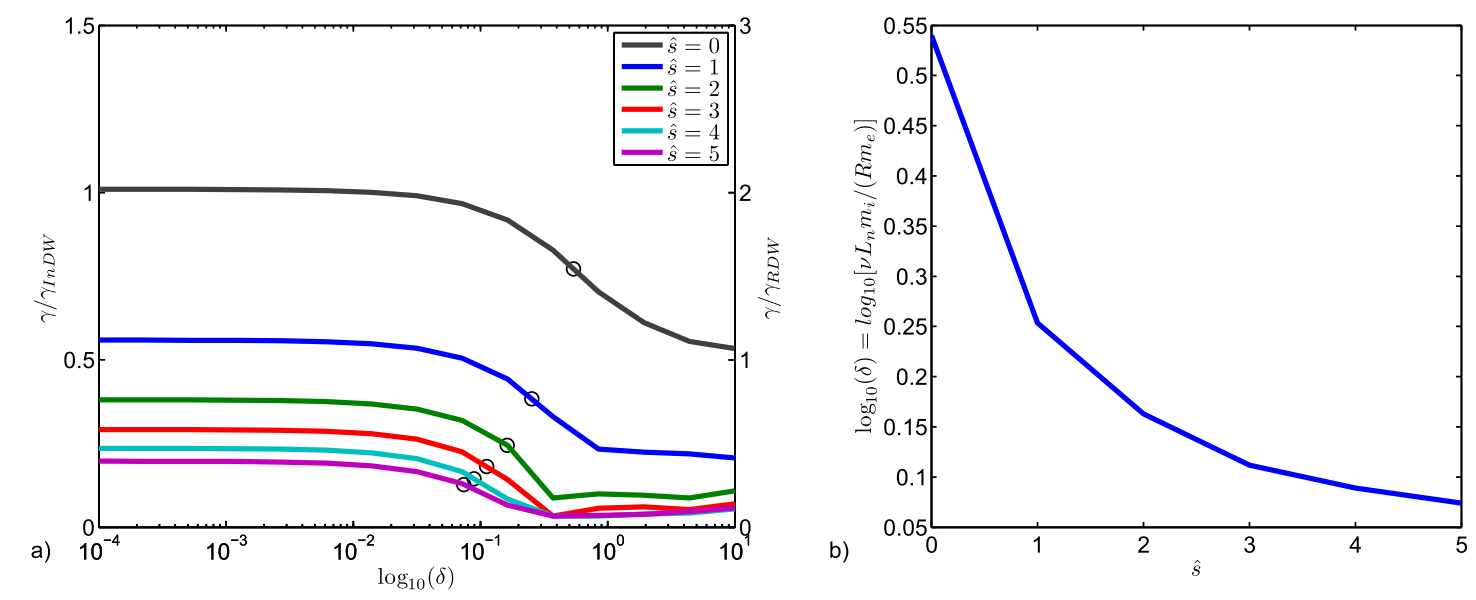

FIG. 9. The transition between inertial and resistive drift waves. The drift wave instability growth rate, $\gamma$, solution of Eq. (13) is plotted as a function of $\delta=\nu L_{n} m_{i} /\left(R m_{e}\right)$ and $\hat{s}$ (a) and the value of $\delta$ at the transition is plotted as a function of $\hat{s}$ (b) [in (a) the bullets indicate the threshold between the two modes]. 


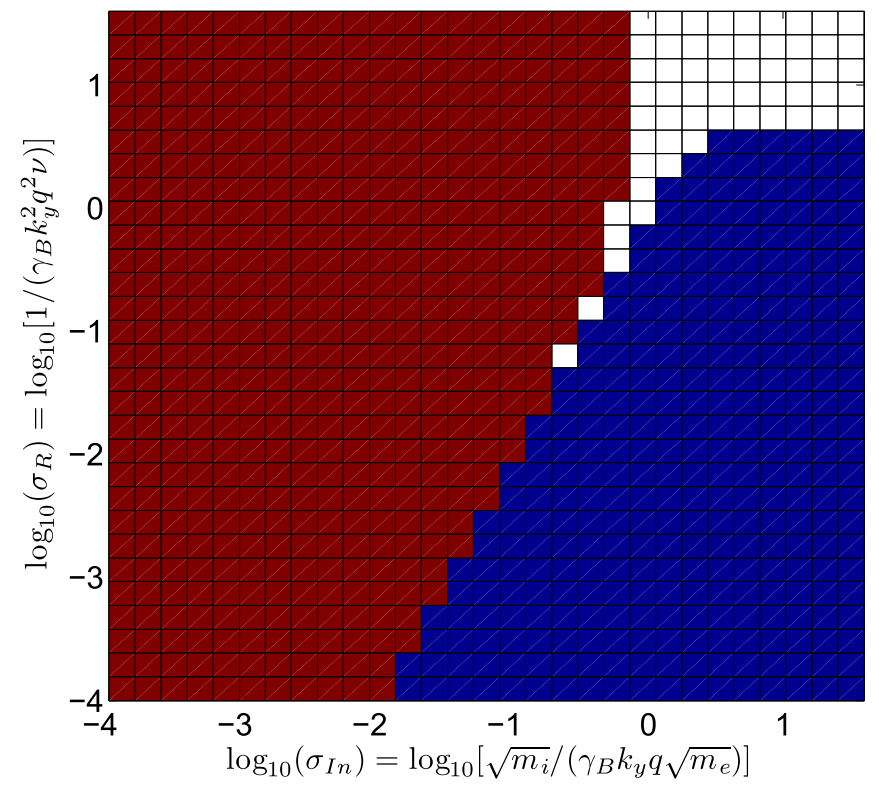

FIG. 10. The transition between resistive and inertial ballooning modes. The ratio $\gamma_{I n B M} / \gamma_{R B M}$ between the growth rate of RBM, solution of Eq. (6), and of the InBM, solution of Eq. (9), is plotted as a function of $\sigma_{I n}$ and $\sigma_{R}$; in the red area, $\gamma_{I n B M} / \gamma_{R B M}>1$, in the blue area, $\gamma_{I n B M} / \gamma_{R B M}<1$, while the white area shows the region where the ratio depends on $\hat{s}$.

Fig. 10, the red surface identifies the region in which the ratio $\gamma_{I n B M} / \gamma_{R B M}$ is bigger than 1 for all values of $\hat{s}$, i.e., the InBM prevails, while the blue surfaces identify the region where the ratio $\gamma_{I n B M} / \gamma_{R B M}$ is smaller than 1, i.e., the RBM prevails, independently of $\hat{s}$. The narrow regions of the plane $\left(\sigma_{R}, \sigma_{I}\right)$ in which the threshold depends on $\hat{s}$ are colored in white. The value of $\sigma_{R}$ for which we observe the transition depends on $\sigma_{I n}$ as $\sigma_{R} \simeq 0.56 \sigma_{I n}^{1.82}$, which provides, therefore, a simple estimate of the transition between RBM and InBM.

\section{E. The role of electromagnetic effects}

We extend the analysis of the linear instability regime to finite $\beta$ plasmas and therefore we consider the effect of the electromagnetic terms on the system of Eqs. (1). Two main phenomena are observed related to finite $\beta$ : suppression of the DW instability, and the appearance of the IdBM, when the ideal limit is overcome.

In order to describe the effect of the electromagnetic terms, the simplest model to consider consists of the system of Eqs. (1), excluding the coupling with sound waves, i.e., $k_{\|} \ll \gamma$ and analyzing the resistive $\left(m_{e} / m_{i}=0\right)$ and inertial $(\nu=0)$ limits. The system can be reduced to the following eigenvalue equation for $\Phi$ :

$$
\begin{aligned}
\gamma k_{\perp}^{2} \Phi= & -\hat{C}\left\{A_{0}+\frac{1}{A_{1}}\left[A_{0}\left(\frac{\hat{C}}{1.71}-1\right)+A_{2}\right]\right\} \Phi \\
& +\frac{k_{\perp}^{2}}{A_{3}}\left\{1-A_{0}-\frac{1.71}{A_{1}}\left[A_{0}\left(\frac{\hat{C}}{1.71}-1\right)+A_{2}\right]\right\} \frac{\partial^{2} \Phi}{\partial \hat{z}^{2}},
\end{aligned}
$$

where $A_{0}=R i k_{y} /\left(\gamma L_{n}\right)-k_{\perp}^{2}-\hat{C} / \gamma, A_{1}=0.88 \gamma-2.05+\hat{C}$, and $A_{3}=\gamma X+k_{\perp}^{2}+k_{\perp}^{2} X(1+1.71 \eta)$. In the resistive case,
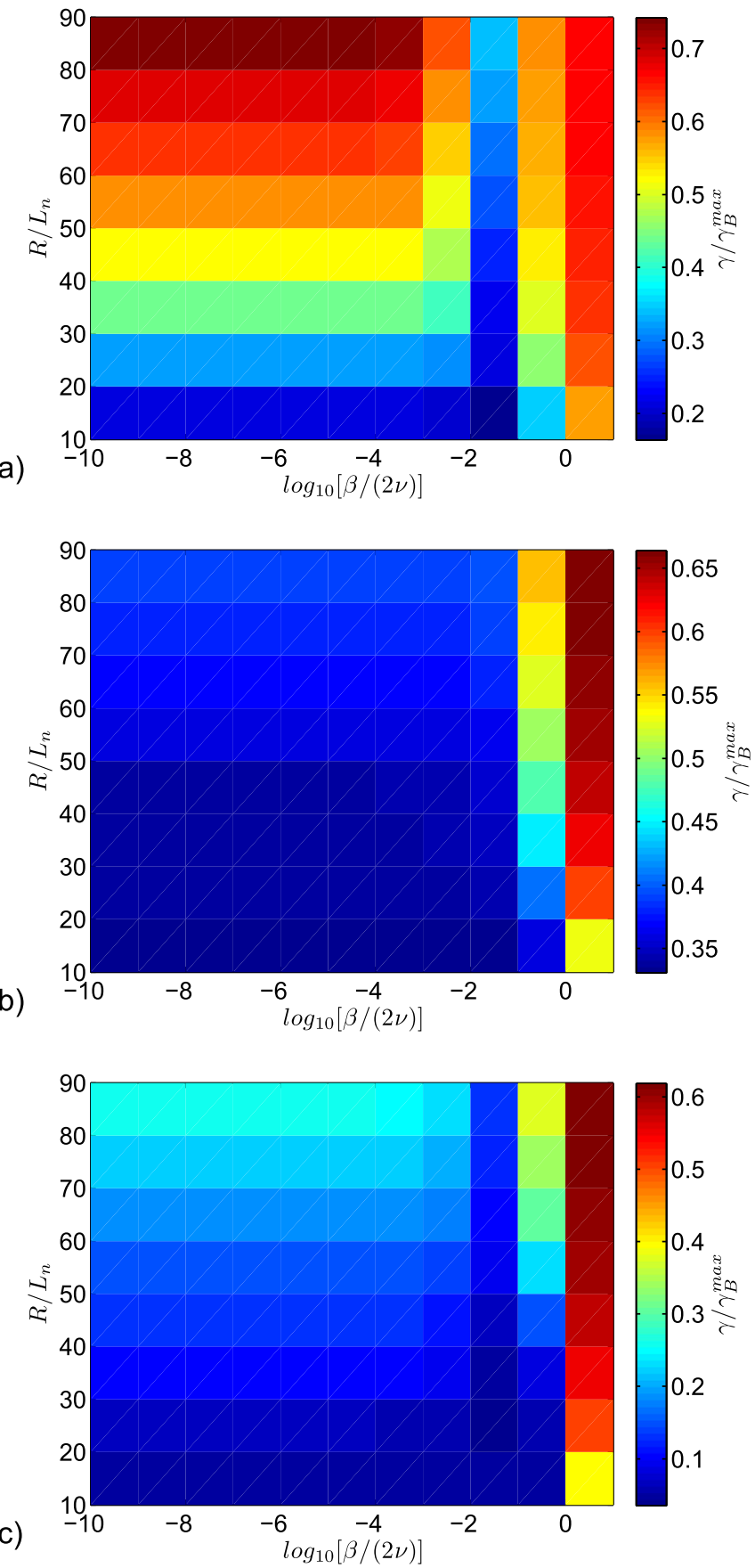

FIG. 11. Role of electromagnetic effects on the resistive instabilities. The normalized growth rate $\gamma / \gamma_{B}^{\max }$, solution of Eq. (16), is plotted as a function of $\beta /(2 \nu)$ and $R / L_{n}$ for $\hat{s}=0$ (a), $\hat{s}=1$ (b), and $\hat{s}=-1$ (c).

$X=\beta /(2 \nu)$ and $0<\hat{z}<2 \pi q \sqrt{\nu}$, while in the inertial case $X=\beta m_{i} /\left(2 m_{e}\right)$ and $0<\hat{z}<2 \pi q \sqrt{m_{e} / m_{i}}$.

In order to illustrate the role of electromagnetic effects, we consider two specific cases, which reflect the typical impact of $\beta \neq 0$ on the instabilities. The maximum growth rate of the instability, solution of Eq. (16), is plotted in Fig. 11 in the resistive limit for $\nu=0.01$ and $q=4$, and in Fig. 12, in the inertial limit, for $m_{e} / m_{i}=2.72 \times 10^{-4}$ and $q=4$. In both cases, $\eta=1$. Focusing on the resistive case, a number of observations can be made. For $\hat{s}=0$ [Fig. 11(a)], at high values of $R / L_{n}$, the RDW is suppressed. As it will be demonstated in the following, this occurs for $\beta /(2 \nu) \simeq 1.17 L_{n} /[R(1+171 \eta)]$. 
a)

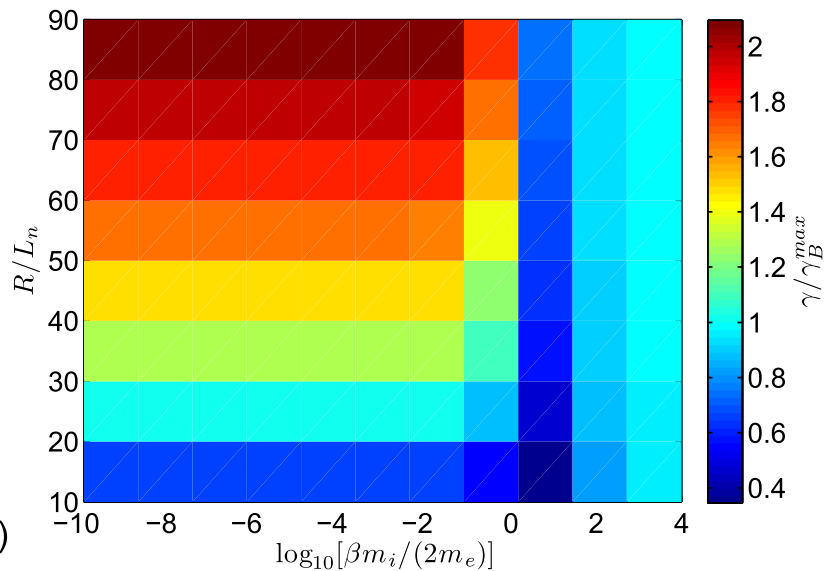

b)

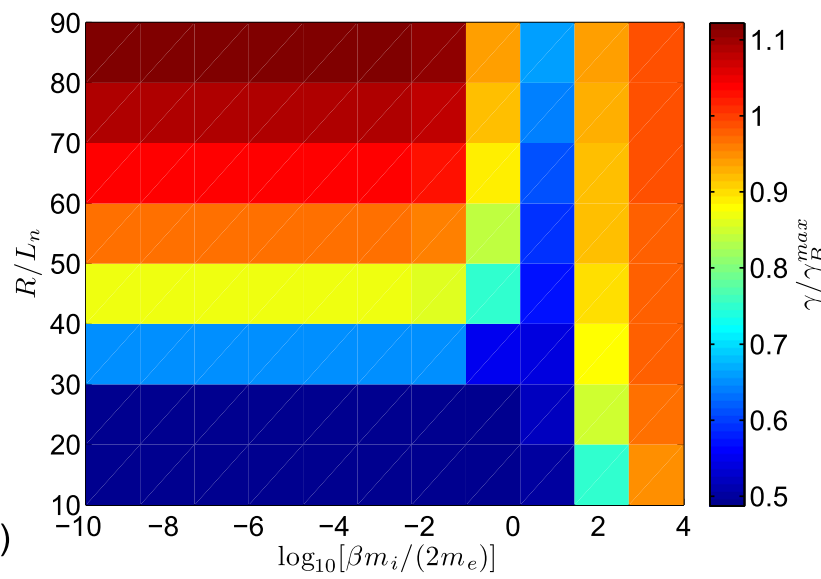

C)

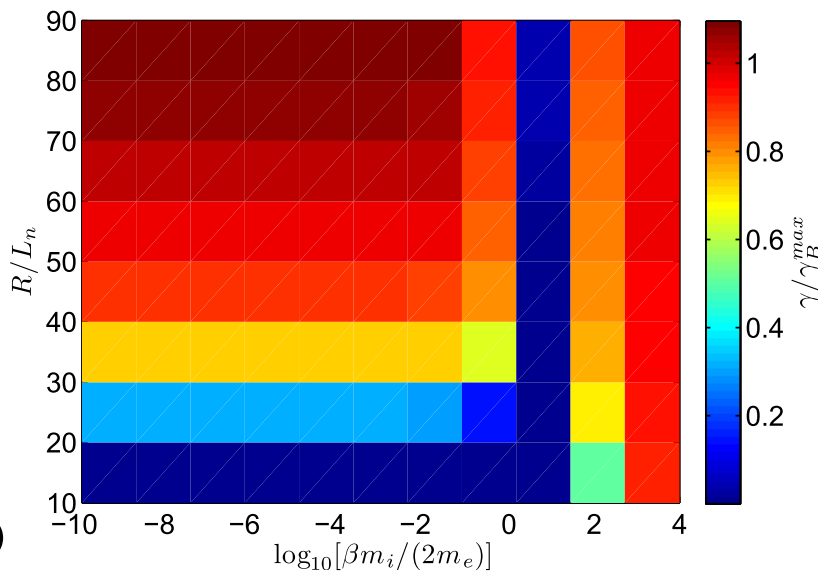

FIG. 12. Role of electromagnetic effects on the inertial case. The normalized growth rate $\gamma / \gamma_{B}^{\max }$, solution of Eq. (16), is plotted as a function of $\beta m_{i} /\left(2 m_{e}\right)$ and $R / L_{n}$ for $\hat{s}=0(\mathrm{a}), \hat{s}=1(\mathrm{~b})$, and $\hat{s}=-1$ (c).

We also observe the appearance of the IdBM instability, once the $\alpha_{M H D}$ threshold is overcome. Since $\alpha_{M H D}$ is proportional to $\beta R / L_{n}$, the $\beta$ threshold for IdBM is inversely proportional to $R / L_{n}$, i.e., the IdBM develops at lower $\beta$ for higher values of $R / L_{n}$. For $\hat{s} \neq 0$, the suppression of the RDW and the appearance of the IdBM is also observed [see Figs. 11(b) and 11(c), which consider $\hat{s}=1$ and $\hat{s}=-1$, respectively]. With respect to the $\hat{s}=0$ case, we also point out: (i) a reduction of the RDW growth rate (high $R / L_{n}$ ) to half of the shearless value, as expected from Fig. 4; (ii) an increase of the RBM growth rate for $\hat{s}=1$ and a decrease for $\hat{s}=-1$ with respect to the shearless value, as expected from Fig. 1; (iii) an increase of the
IdBM growth rate for $\hat{s}=1$ and a decrease for $\hat{s}=-1$ with respect to the shearless value, as expected from Fig. 2. We finally note that for $\hat{s}<0$, IdBM is less suppressed by magnetic shear than RBM. This is due to the fact that for the characteristic values of $\alpha_{M H D}$ in Fig. 11, the $\hat{s}$ damping is minimum: for example, for $\beta=2 \times 10^{-3}, R / L_{n}=50$, we have $\alpha_{M H D}=3.2$, consequently the mode is highly unstable for any value of the magnetic shear (see Fig. 3). On the other hand, we are considering the RBM instability at high values of $\sigma_{R}$ where the dependence of the growth rate on the shear is more evident: for the same set of parameters $\sigma_{R}=1.77$ and $\hat{s}$ strongly reduces the growth rate (see Fig. 1).

In the inertial case (Fig. 12), similar observations as in the resistive case can be made. For $\hat{s}=0$ [see Fig. 12(a)], at high values of $R / L_{n}$, the InDW instability is dominant for $\beta m_{i} /\left(2 m_{e}\right) \geq 0.17$, as it is shown in the following. We also observe the appearance of the IdBM instability, at $\beta$ value that is inversely proportional to $R / L_{n}$. For $\hat{s}= \pm 1$, the remarks made for the resistive case remain valid.

Now, we analyze in details the suppression of the DW instability due to the electromagnetic effects by considering a relatively simple model. We reduce the system of Eqs. (13) to an algebraic dispersion relation by considering the $\hat{s}=0$ case and substituting $\partial / \partial z \rightarrow i k_{\|}$, and we consider electromagnetic effects acting on both the InDW (by setting $\nu=0$ ) and the RDW (with $m_{e} / m_{i}=0$ ). Within these hypothesis, the dispersion relation has the form $\bar{\gamma}^{3} b_{3}+\bar{\gamma}^{2} b_{2}+\bar{\gamma} b_{1}+b_{0}=0$, where $\bar{\gamma}=\gamma /\left[(1+171 \eta) R / L_{n}\right]$. In the resistive case, the coefficients in the dispersion relation are: $b_{3}=-i X, b_{2}=i k_{y}^{2}+X k_{y}$, $b_{1}=i Z^{2}\left[(1+295) k_{y}^{2}\right], \quad b_{0}=Z^{2} k_{y}, \quad$ being $\quad X=(1+$ $1.71 \eta) \beta R /\left(2 \nu L_{n}\right)$ and $Z=k_{\|} \sqrt{L_{n}} / \sqrt{\nu R(1+1.71 \eta)}$. In Fig. 13(a), we show the maximum growth rate over $k_{y}$ and $k_{\|}$ as a function of $X$. Numerically, we verify that the growth rate is reduced to half of the maximum for $X>1.17$, i.e., the RDW is suppressed by electromagnetic effects for $\beta>2.34 \nu L_{n} /[R(1+1.71 \eta)]$. On the other hand, in the inertial case, $b_{3}=i k_{y}^{2}+i X, \quad b_{2}=X k_{y}, \quad b_{1}=i Z^{2}\left(1+295 k_{y}^{2}\right)$, $b_{0}=Z^{2} k_{y}$, with $X=\beta m_{i} /\left(2 m_{e}\right) \quad$ and $\left.\quad Z=k_{\|} L_{n} \sqrt{m_{i}}\right)$ $\left[R \sqrt{m_{e}}(1+1.71 \eta)\right]$. In Fig. 13(b), we show the maximum growth rate over $k_{y}$ and $k_{\|}$as a function of $X$ : the growth rate is reduced to the half of the maximum for $X>0.17$, i.e., the InDW is suppressed for $\beta>0.34 m_{e} / m_{i}$.

To summarize, with the introduction of electromagnetic effects, we observe two main phenomena in our system. At high values of $R / L_{n}$, the RDW and the InDW are suppressed at $\beta>$ $2.34 \nu L_{n} /[R(1+1.71 \eta)]$ and $\beta>0.34 m_{e} / m_{i}$, respectively. When the $\alpha_{M H D}$ threshold is overcome, then the IdBM starts to play a role and we expect the shift of the fastest growing instability from finite $k_{y}$ values to the smallest allowed $k_{y}$ value.

\section{EXAMPLES OF LINEAR STABILITY ANALYSIS}

In this section, we use the framework built in Sec. V to identify and analyse the linear instability present in three typical SOL scenarios. For this purpose, we use a linear code that solves the system of Eqs. (1) as a function of the toroidal mode number $n$ (see Appendix A for details) and we identify the dominant instability according to our parameter space, testing the reliability of our analysis by exploring the 

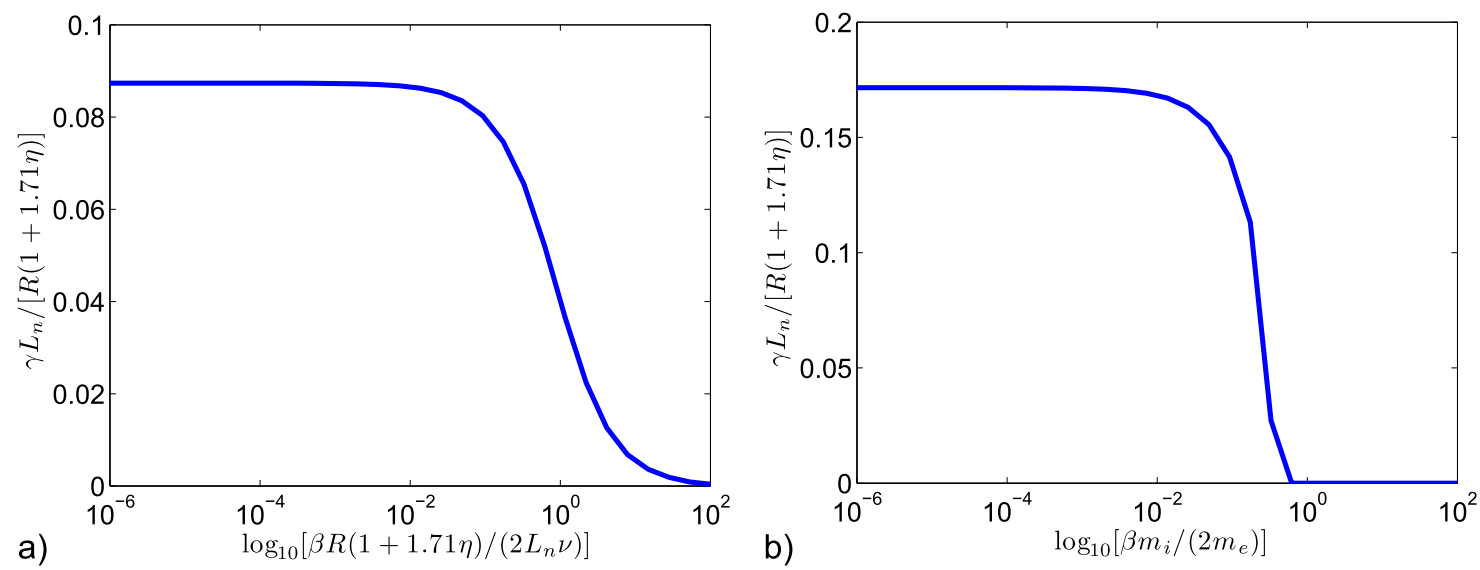

FIG. 13. Role of electromagnetic effects on the drift waves: suppression of the drift waves growth rate in the resistive limit (a) and in the inertial limit (b) for increasing $\beta$.

dependence of the instability on $\hat{s}$ and $\beta$. We focus our attention on the following sets of parameters: first, a parameter set with $R / L_{n}=10, \quad L_{y}=1000, q=4, \quad \nu=0.1$, and $m_{e} / m_{i}=2.72 \times 10^{-4}$, called "low-gradient;" second, a "high-gradient" parameter set, with $R / L_{n}=90, \nu=0.01$, being the other parameters the same as in the first set; third, we apply our analysis to a TCV tokamak ${ }^{45}$ L-mode discharge, where the plasma with approximately circular flux surfaces is created close to the high-field side of the machine, creating a scenario that reproduces the toroidal limiter configuration considered here: $R / L_{n}=25, \quad R / L_{T}=35$ $L_{y}=1610, R=1025, q=3, \nu=3.16 \times 10^{-3}$, and $m_{e} / m_{i}$ $=2.72 \times 10^{-4}$. The parameter sets used are summarized in Table I.

We first consider the low-gradient set of parameters. Our analysis indicates that the SOL corresponding to this parameter set is in the $\mathrm{BM}$ dominated regime. In fact, $R / L_{n}$ is smaller than the threshold value between RBM and RDW, as calculated in Sec. V A, and it is also smaller than the threshold between InBM and InDW, as calculated in Sec. V B. Moreover, according to the results shown in Fig. 10, since $\sigma_{R} \simeq 0.44$ and $\sigma_{I} \simeq 5.25$, the instability belongs to the resistive branch of the BM. We first consider the effect of $\hat{s}$ on the instability. In Fig. 14, we show $\gamma$ as a function of $n$, for different value of the magnetic shear, in the $\beta=0$ limit. Our analysis (see Sec. III A) shows that the maximum expected growth rate is for $1 /\left(2 \pi q \sqrt{\gamma_{B}^{\max } \nu}\right)<k_{y}<0.3 \gamma_{B}^{\max }$, which in our case corresponds to $0.052<k_{y}<1.73$, therefore, the peak growth rate is expected at $k_{y} \simeq 1$. Since $k_{\|} \ll k_{y}$, we can estimate the toroidal mode number as $n \simeq m / q$, where $m$ is the poloidal mode number, thus the interval can also be expressed as $2<n<69$. Effectively, the results of the linear

TABLE I. List of the parameters for the three cases analyzed in the linear stability analysis. The TCV L-mode parameter set reflects the equilibrium of shot no. 42237.

\begin{tabular}{lcccccc}
\hline \hline Name & $2 \pi a$ & $q$ & $\nu$ & $m_{e} / m_{i}$ & $R / L_{n}$ & $\eta$ \\
\hline Low-gradient & 1000 & 4 & 0.1 & $2.72 \times 10^{-4}$ & 10 & 1 \\
High-gradient & 1000 & 4 & 0.01 & $2.72 \times 10^{-4}$ & 90 & 1 \\
TCV L-mode & 1610 & 3 & $3.16 \times 10^{-3}$ & $2.72 \times 10^{-4}$ & 25 & 0.71 \\
\hline \hline
\end{tabular}

code shows that the maximum growth rate, $\gamma \simeq 0.53 \gamma_{B}^{\max }$, is reached for $k_{y} \simeq 0.50$, which corresponds to a toroidal mode number $n \simeq 20$, in agreement with our estimate. We also observe, as expected from the analysis in Sec. III A, the maximum of the growth rate for $\hat{s} \simeq 1$ (see Fig. 1). The influence of electromagnetic effects is studied in Fig. 15, where we show $\gamma$ as a function of $n$ for different values of $\beta$. We verify the development of the IdBM when the ideal threshold is overcome. At $\hat{s}=0$, the IdBM growth rate rises up to $0.5 \gamma_{B}^{\max }$ when $\alpha_{M H D} \simeq 0.58$, according to the results shown in Fig. 3, and consequently the limit for the development of the IdBM is overcome when $\beta>1.8 \times 10^{-3}$. According to our observations, we remark a shift of the maximum growth rate from finite $k_{y}$ towards $k_{y} \rightarrow 0$, typical of the IdBM instability, at the expected $\beta$ threshold.

Considering the high-gradient parameter set, from the analysis in Sec. V, we conclude that it falls in the parameter space region where the RDW is the fastest growing instability. In fact, $R / L_{n}$ is above both the threshold between $\mathrm{RBM}$ and RDW (see Sec. V A) and the threshold between InBM and InDW (see Sec. V B). Moreover, according to the results

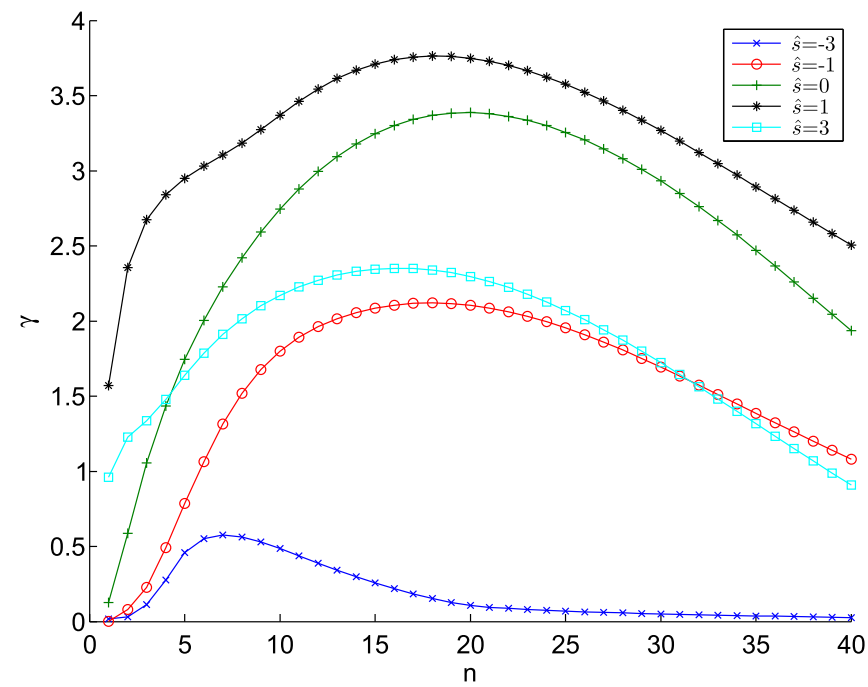

FIG. 14. Linear growth rate $\gamma$, solution of Eqs. (1), as a function of the toroidal mode number $n$, for different values of $\hat{s}$, for the "low-gradient" set of parameters. 


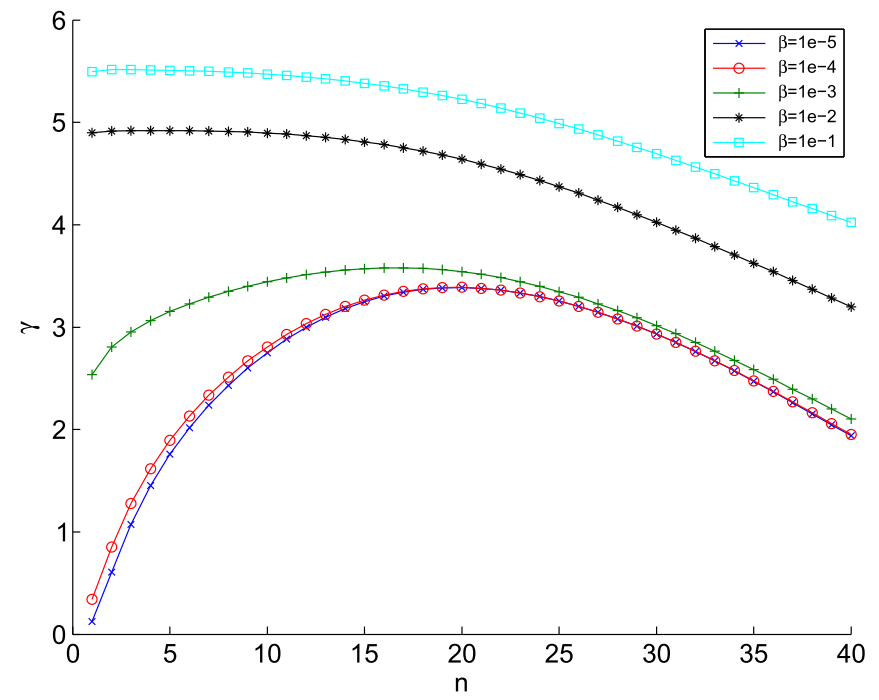

FIG. 15. Linear growth rate $\gamma$, solution of Eqs. (1), as a function of the toroidal mode number $n$, for different values of $\beta$, for the "low-gradient" set of parameters.

shown in Fig. 9, for $\delta \simeq 0.41(\gamma \simeq 34.20)$, the DW is in the parameter range of the resistive branch, although marginal influence by inertial effects may be expected. In Fig. 16, we show $\gamma$ as a function of $n$, for different values of $\hat{s}$, as calculated by the linear code. The maximum growth rate is $\gamma \simeq 32.15$, while, with the considered parameters $\gamma_{R D W}^{\max } \simeq 20.73$. The difference is due to the presence of inertial effects, which increases the growth rate with respect to the purely resistive case. The analysis in Sec. IV A shows that the peak growth rate is expected at $k_{y} \simeq 0.57$ and, since $k_{\|} \ll k_{y}$, corresponding to $n \simeq m / q \simeq 22$. The linear code confirms that the maximum growth rate is reached at $k_{y} \simeq$ 0.57 and $n \simeq 22$, close to the poloidal and toroidal mode number estimate. The peak growth rate is observed at $\hat{s}=0$ and, for both $\hat{s}>0$ and $\hat{s}<0$, we remark a decrease of the growth rate, according to the results in Fig. 4. We underline that in the linear code, the curvature term is retained in all

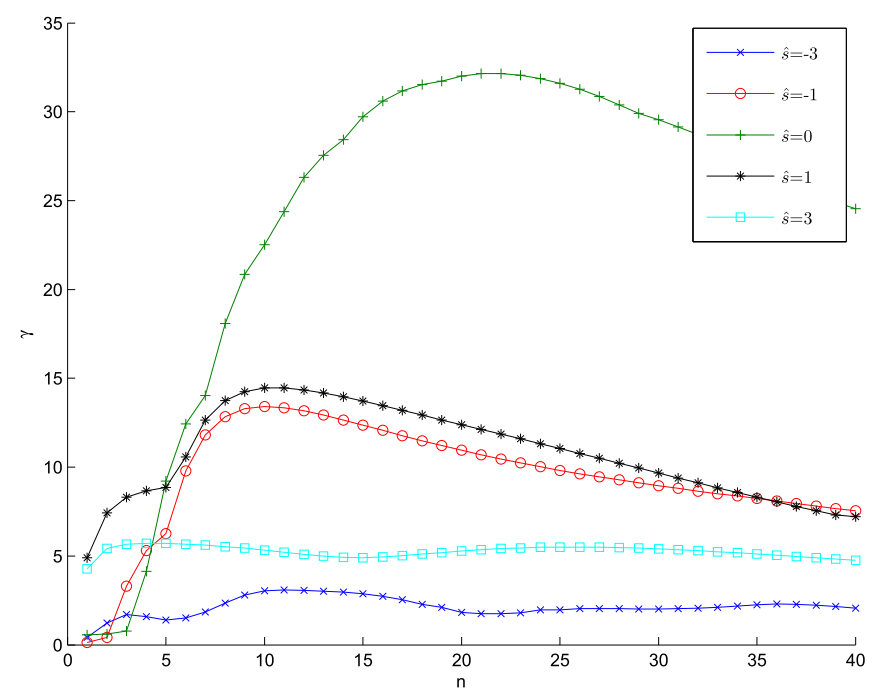

FIG. 16. Linear growth rate $\gamma$, solution of Eqs. (1), as a function of the toroidal mode number $n$, for different values of $\hat{s}$, for the "high-gradient" set of parameters. the equations, while in the simplified fluxtube model used to compute the results in Fig. 4, it is neglected. This introduces an asymmetric behaviour of the solutions with respect to $\hat{s}>0$ and $\hat{s}<0$. We also analyze electromagnetic effects on the RDW instability that we are considering here. In Fig. 17, we show $\gamma$ as a function of $n$, for different values of $\beta$. For $\beta=1 \times 10^{-4}$, we observe that the growth rate decreases to about $1 / 3$ of the maximum value obtained for $\beta=1 \times 10^{-5}$. This is due to the electromagnetic damping of the RDW. The effect starts to be noticeable for $\beta>2.34 L_{n} \nu /[R(1$ $+171 \eta)] \simeq 9.59 \times 10^{-5}$, according to Sec. VE. Since, for the parameters under consideration, the $\alpha_{M H D}$ limit for the IdBM is overcome when $\beta \gtrsim 2.01 \times 10^{-4}$, we note that there is a window of $\beta$ values in which the RDW instability is suppressed and the IdBM is not unstable. For $\beta=1 \times 10^{-3}$, we observe the appearance of the IdBM instability, where we note the shift of the maximum growth rate from finite $k_{y}$ to $k_{y} \rightarrow 0$, as expected for the IdBM instability. The maximum growth rate decreases to $\gamma \simeq \gamma_{B}^{\max } \simeq 18.52$, close to the maximum growth rate of $\mathrm{BM}$ instabilities.

Finally, we analyze the L-mode discharge in the TCV tokamak. We find that, according to the parameter space analysis, the SOL of this configuration is in the InDW region. In fact, for this set of parameters, we evaluate $\delta \simeq 0.46, \sigma_{R} \simeq 20.54$, and $\sigma_{I n} \simeq 5.10$, therefore, inertial effects partially dominate over resistive effects, as shown in Fig. 9. Moreover, since $R / L_{n} \simeq 25$, we are in the regime where the DW grow faster. The highest growth rate of $\gamma=10.58$ is reached at $n=39$ and $m=112$, corresponding to $k_{y}=0.44$; for comparison, we note that the maximum InDW growth rate for the considered parameters is $\gamma_{\operatorname{In} D W}^{\max }$ $=9.44$ at $k_{y}=0.57$. The nature of the instability changes with $\hat{s}$. In fact, at $\hat{s}=2$, the InBM prevails, with the maximum growth rate of $\gamma=3.42$ at $n=13$ and $m=40$, corresponding to $k_{y}=0.16$. At this $k_{y}$ value, $\sigma_{I n}=43.06$, causing a damping of the growth rate for InBM to $\gamma \simeq 0.3 \gamma_{B}^{\max }$ $\simeq 2.78$, according to the parameter space analysis. At $\hat{s}=-2$, both the InBM and the InDW are suppressed.

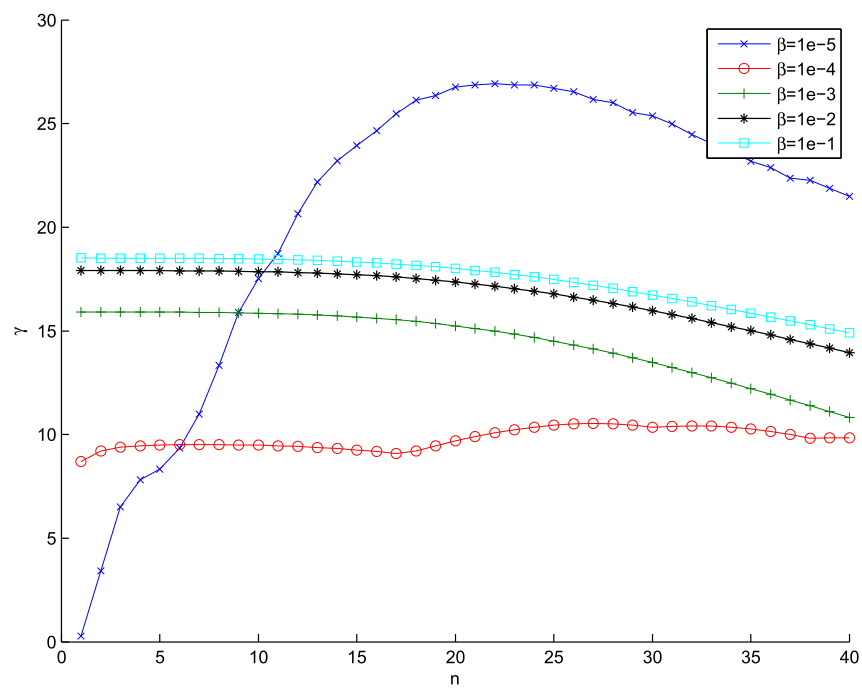

FIG. 17. Linear growth rate $\gamma$, solution of Eqs. (1), as a function of the toroidal mode number $n$, for different values of $\beta$, for the "high-gradient" set of parameters. 


\section{CONCLUSIONS AND DISCUSSION}

The present paper provides a framework to identify the fastest growing instabilities as a function of the parameters characterizing the tokamak SOL region. We have considered the local, linearized, drift-reduced Braginskii equations with cold ions, in the infinite aspect ratio SOL geometry with a toroidal limiter. We have identified the regimes of linear instabilities due to the presence of the resistive and inertial branches of the DW and the resistive, inertial, and ideal branches of the BM. Starting from a detailed analysis of each instability, we have identified the boundaries of the SOL parameter space regions dominated by each mode.

In the electrostatic limit, we observe that DW dominates over the BM at steep gradients. In general, the $R / L_{n}$ threshold depends on $\sigma_{R}=1 /\left(\gamma_{B}^{\max } k_{y}^{2} q^{2} \nu\right)$, $\sigma_{I n}=\sqrt{m_{i}} /\left(\gamma_{B}^{\max } k_{y} q \sqrt{m_{e}}\right)$, and $\hat{s}$ (Figs. 7 and 8). The transition between RDW and InDW is governed by the $\delta=$ $\nu L_{n} m_{i} /\left(R m_{e}\right)$ parameter and it occurs at $\delta \simeq 3.55$ for $\hat{s}=0$, with the transition value of $\delta$ decreasing with the increase of $|\hat{s}|$ (Fig. 9). The regions of influence of the RBM and the InBM have been evaluated as a function of $\sigma_{R}$ and $\sigma_{I n}$, the boundary between those is independent of $\hat{s}$ for most of the values of $\sigma_{R}$ and $\sigma_{I n}$ and the transition occurs for $\sigma_{R} \simeq 0.56 \times \sigma_{I n}^{1.82}$ (Fig. 10). Electromagnetic effects cause, at high $R / L_{n}$, the damping of the DW instability at $\beta /(2 \nu) \simeq 1.17 L_{n} /[R(1+171 \eta)]$ in the resistive case, and at $\beta m_{i} /\left(2 m_{e}\right) \simeq 0.17$ in the inertial case (Fig. 13). The appearance of the IdBM instability is observed when the $\alpha_{M H D}=q^{2} \beta(1+\eta) R / L_{n}$ threshold is overcome (Figs. 11 and 12).

We have used our framework to interpret the results of a linear code that evaluates the growth rate of the SOL instabilities. By considering three different sets of SOL parameters, we have identified the main instability governing the physical system in each scenario, showing that we can predict the dependence of each instability on magnetic shear and plasma $\beta$.

We notice that in existing tokamaks $R / L_{n}$ spans one order of magnitude and $\nu$ two orders of magnitude (see, e.g., Refs. 24-32). Both DW and BM instabilities can exist in this range of $R / L_{n}$ and both resistive and inertial effects are important, and therefore we expect the behaviour of the SOL to change remarkably in these wide intervals of parameters. Our parameter space analysis has been conceived as a first stage tool to be used in the understanding of turbulence in the SOL of tokamaks, necessary to interpret the results of non-linear simulations.

\section{ACKNOWLEDGMENTS}

This work is supported by the Swiss National Science Foundation. We acknowledge useful discussions with $\mathrm{F}$. Avino, S. Coda, I. Furno, B. Rogers, O. Sauter, and D. Wagner.

\section{APPENDIX: NUMERICAL SCHEMES}

We describe the code used to evaluate the growth rate of the SOL instability by solving the eigenvalue problem in Eqs. (1). The unknowns are the perturbed density $n$, electrostatic potential $\Phi$, magnetic flux $\psi$, electron temperature $T_{e}$, and ion parallel velocity $V_{\| i}$. We assume perturbations in the form $f_{n}(y, z, t)=f_{n}(y) \exp (i n \phi+\gamma t)$, where $n$ is the toroidal mode number, and $\phi$ is the toroidal angle. We discretize $y=$ $\left[0, L_{y}\right]$ with $N_{y}$ points, $y_{1}, \ldots, y_{i}, \ldots, y_{N_{y}}$, with $y_{i}=(i-1)$ $L_{y} /\left(N_{y}-1\right)$ and we evaluate $n, \Phi$, and $T_{e}$ at these points. The quantities $\psi$ and $V_{\| i}$ are evaluated on $N_{y}-1$ points, $y_{1}, \ldots, y_{i}, \ldots, y_{N_{y}-1}$, with $y_{i}=(i-1 / 2) L_{y} /\left(N_{y}-1\right)$ for $\psi$ and $V_{\| i}$. We denote the grid on which we evaluate $n$, $\Phi$, and $T_{e}$ as the unshifted grid, while the grid for $\psi$ and $V_{\| i}$ is referred to as the shifted grid. We also denote $\Delta_{y}=L_{y} /\left(N_{y}-1\right)$. We introduce the vector $\vec{x}=\left[n_{1}, \ldots, n_{N_{y}}\right.$, $\left.\Phi_{1}, \ldots \Phi_{N_{y}}, T_{e, 1}, \ldots, T_{e, N_{y}}, \psi_{1}, \ldots, \psi_{N_{y}-1}, V_{\| i, 1}, \ldots, V_{\| i, N_{y}-1}\right]$, and rewrite Eqs. (1) as

$$
L \frac{\partial}{\partial t} \vec{x}=M \vec{x}
$$

where

$$
L=\left(\begin{array}{ccccc}
U_{u} & Z_{u, u} & Z_{s, u} & Z_{u, u} & Z_{s, u} \\
Z_{u, u} & D_{u, u}^{y, 2} & Z_{s, u} & Z_{u, u} & Z_{s, u} \\
Z_{u, s} & Z_{u, s} & -m_{e} / m_{i} D_{s, s}^{y, 2}-\beta / 2 & Z_{u, s} & Z_{s, s} \\
Z_{u, u} & Z_{u, u} & Z_{s, u} & U_{u} & Z_{s, u} \\
Z_{u, s} & Z_{u, s} & Z_{s, s} & Z_{u, s} & U_{s}
\end{array}\right),
$$

and

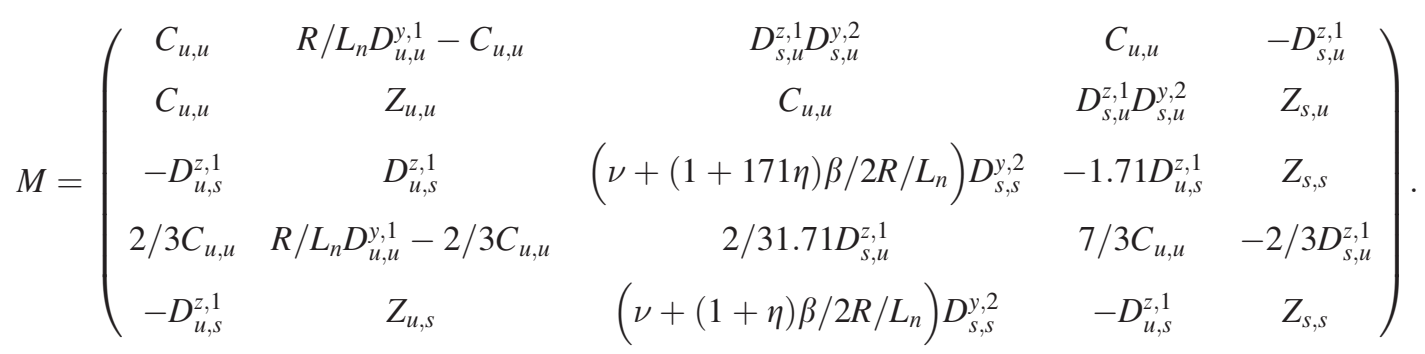


We note that $U$ is the identity matrix, $Z$ is the empty matrix while the $D$ matrices are discretized differential operators for which the first superscript indicates the variable with respect to which the derivative is calculated, the second superscript indicates the order of the derivative. For every matrix, the first subscript indicates the shifted (s) or unshifted (u) grid on which the operator is acting, the second subscript indicates the grid type or the resulting variable. Both $D_{u, u}$ and $D_{s, s}$ are square matrixes, the first with $N_{y} \times N_{y}$ dimensions and the second with $\left(N_{y}-1\right)$ $\times\left(N_{y}-1\right)$ dimensions. The generic differential operators are written as

$$
D^{k, p} B_{i}=\left.\frac{\partial^{k}}{\partial y^{k}}\right|_{y=y_{i}} \simeq \frac{1}{(\Delta y)^{k}} \sum_{n=-p / 2}^{p / 2} A_{n}^{k, p} B_{i+n},
$$

where $p$ is the accuracy order of the scheme. Coefficients for $D_{u, u}$ and $D_{s, s}$ are similar. Coefficients $A_{k}^{n}$ are obtained by Taylor expanding $B_{i+n}=B\left(y_{i+n}\right)$ around $y_{i}$. Coefficients for $D_{u, s}$ and $D_{s, u}$ are obtained in a similar way by replacing $i$ by $i+1 / 2$. The $C$ matrix is the curvature operator, constructed by combining the appropriate differential operators defined above, according to Eq. (2). We remark that the parallel derivative is calculated as $\partial f_{n} / \partial z=a / q \partial f_{n} / \partial y$ $+\inf f_{n}$.

The eigenvalue problem is solved using three different approaches. The first one is the direct solution of the problem associated to Eq. (A1), providing the whole spectrum of eigenmodes and eigenvalues of the system. This was accomplished by using the LAPACK library. ${ }^{46}$ The second method is an iterative solver that integrate the time evolution of the system (A1) by discretizing it with an implicit scheme in the form

$$
\frac{\vec{x}_{t+\Delta t}-\vec{x}_{t}}{\Delta t}=(1-\Theta) L^{-1} M \vec{x}_{t}+\Theta L^{-1} M \vec{x}_{t+\Delta t},
$$

where the choice of $\Theta=0$ leads to a fully explicit scheme, while $\Theta \neq 0$ leads to a semi-implicit scheme. The growth rate is calculated by comparing the solution at two different time steps. The third approach is based on considering the time evolution of the system (A1) and evaluating the exponential of the matrix $L^{-1} M \Delta t$, having fixed a desidered time step $\Delta t$. The employed method is the Padé approximation described in Ref. 47 The growth rate can be calculated comparing the solution at two different time steps. The calculation of the exponential matrix is costly, but the successive iterations are extremely fast. The iterative solver is usually faster than the other two methods. We have verified that the three methods, applied to the same set of parameters, give similar results. For the linear global calculations presented in this article, we use the spectral solver with a fourth order finite difference scheme.

The differential operators are discretized differently in the fluxtube code used to evaluate the linear growth rates of Sec. V. In the fluxtube code each perturbed quantity is Fourier decomposed in the $y$ direction: $f(y, z, t) \propto \exp$ $\left(i k_{y} y+\gamma t\right)$. The parallel derivative is discretized with a second order finite difference scheme.
${ }^{1}$ E. Doyle, W. Houlberg, Y. Kamada, V. Mukhovatov, T. Osborne, A. Polevoi, G. Bateman, J. Connor, J. Cordey, T. Fujita, X. Garbet, T. Hahm, L. Horton, A. Hubbard, F. Imbeaux, F. Jenko, J. Kinsey, Y. Kishimoto, J. Li, T. Luce, Y. Martin, M. Ossipenko, V. Parail, A. Peeters, T. Rhodes, J. Rice, C. Roach, V. Rozhansky, F. Ryter, G. Saibene, R. Sartori, A. Sips, J. Snipes, M. Sugihara, E. Synakowski, H. Takenaga, T. Takizuka, K. Thomsen, M. Wade, H. Wilson, ITPA Transport Physics Topical Group, ITPA Confinement Database, Modelling Topical Group, and ITPA Pedestal and Edge, Nucl. Fusion 47, S18 (2007).

${ }^{2}$ A. Loarte, B. Lipschultz, A. Kukushkin, G. Matthews, P. Stangeby, N. Asakura, G. Counsell, G. Federici, A. Kallenbach, K. Krieger, A. Mahdavi, V. Philipps, D. Reiter, J. Roth, J. Strachan, D. Whyte, R. Doerner, T. Eich, W. Fundamenski, A. Herrmann, M. Fenstermacher, P. Ghendrih, M. Groth, A. Kirschner, S. Konoshima, B. LaBombard, P. Lang, A. Leonard, P. Monier-Garbet, R. Neu, H. Pacher, B. Pegourie, R. Pitts, S. Takamura, J. Terry, E. Tsitrone, ITPA Scrape-off Layer, and Divertor Physics Topical Group, Nucl. Fusion 47, S203 (2007).

${ }^{3}$ B. Scott, Plasma Phys. Controlled Fusion 39, 1635 (1997).

${ }^{4}$ J. F. Drake, A. Zeiler, and D. Biskamp, Phys. Rev. Lett. 75, 4222 (1995).

${ }^{5}$ A. Zeiler, D. Biskamp, J. F. Drake, and P. N. Guzdar, Phys. Plasmas 3, 2951 (1996).

${ }^{6}$ A. Zeiler, Report IPP 5/88, Max-Planck-Institut fur Plasmaphysik, 1999.

${ }^{7}$ J. W. Connor, R. J. Hastie, and J. B. Taylor, Phys. Rev. Lett. 40, 396 (1978).

${ }^{8}$ G. Bateman and D. B. Nelson, Phys. Rev. Lett. 41, 1804 (1978).

${ }^{9}$ D. R. McCarthy, P. N. Guzdar, J. F. Drake, J. T. M. Antonsen, and A. B. Hassam, Phys. Fluids B: Plasma Phys. 4, 1846 (1992).

${ }^{10}$ S. V. Novakovskii, P. N. Guzdar, J. F. Drake, C. S. Liu, and F. L. Waelbroeck, Phys. Plasmas 2, 781 (1995).

${ }^{11}$ T. Rafiq, G. Bateman, A. H. Kritz, and A. Y. Pankin, Phys. Plasmas 17, 082511 (2010).

${ }^{12}$ T. Rafiq, C. C. Hegna, J. D. Callen, and A. H. Kritz, Phys. Plasmas 16, 102505 (2009).

${ }^{13}$ A. Zeiler, D. Biskamp, and J. F. Drake, Phys. Plasmas 3, 3947 (1996).

${ }^{14}$ A. Zeiler, D. Biskamp, J. Drake, and B. Rogers, Phys. Plasmas 5, 2654 (1998).

${ }^{15}$ A. Kendl and B. D. Scott, Phys. Rev. Lett. 90, 035006 (2003).

${ }^{16}$ B. D. Scott, Phys. Plasmas 12, 062314 (2005).

${ }^{17}$ P. Ricci and B. N. Rogers, Phys. Plasmas 16, 092307 (2009).

${ }^{18}$ P. Ricci and B. N. Rogers, Phys. Rev. Lett. 104, 145001 (2010).

${ }^{19}$ K. Mima and A. Hasegawa, Phys. Fluids 21, 81 (1978).

${ }^{20} \mathrm{M}$. Wakatani and A. Hasegawa, Phys. Fluids 27, 611 (1984).

${ }^{21}$ H. Sugama, M. Wakatani, and A. Hasegawa, Phys. Fluids 31, 1601 (1988).

${ }^{22}$ W. Horton, Rev. Mod. Phys. 71, 735 (1999).

${ }^{23}$ P. H. Diamond, A. Hasegawa, and K. Mima, Plasma Phys. Controlled Fusion 53, 124001 (2011).

${ }^{24}$ B. LaBombard, J. W. Hughes, N. Smick, A. Graf, K. Marr, R. McDermott, M. Reinke, M. Greenwald, B. Lipschultz, J. L. Terry, D. G. Whyte, S. J. Zweben, and A. C.-M. Team, Phys. Plasmas 15, 056106 (2008).

${ }^{25}$ O. Garcia, R. Pitts, J. Horacek, A. Nielsen, W. Fundamenski, J. Graves, V. Naulin, and J. J. Rasmussen, J. Nucl. Mater. 363-365, 575 (2007).

${ }^{26}$ V. Mukhovatov, M. Shimada, A. N. Chudnovskiy, A. E. Costley, Y. Gribov, G. Federici, O. Kardaun, A. S. Kukushkin, A. Polevoi, V. D. Pustovitov, Y. Shimomura, T. Sugie, M. Sugihara, and G. Vayakis, Plasma Phys. Controlled Fusion 45, A235 (2003).

${ }^{27}$ C. Gormezano, A. Sips, T. Luce, S. Ide, A. Becoulet, X. Litaudon, A. Isayama, J. Hobirk, M. Wade, T. Oikawa, R. Prater, A. Zvonkov, B. Lloyd, T. Suzuki, E. Barbato, P. Bonoli, C. Phillips, V. Vdovin, E. Joffrin, T. Casper, J. Ferron, D. Mazon, D. Moreau, R. Bundy, C. Kessel, A. Fukuyama, N. Hayashi, F. Imbeaux, M. Murakami, A. Polevoi, and H. S. John, Nucl. Fusion 47, S285 (2007).

${ }^{28}$ Y. Igitkhanov, G. Janeschitz, G. W. Pacher, M. Sugihara, H. D. Pacher, D. E. Post, E. Solano, J. Lingertat, A. Loarte, T. Osborne, O. P. Pogutse, M. Shimada, and W. Suttrop, Plasma Phys. Controlled Fusion 40, 837 (1998).

${ }^{29}$ F. Militello and W. Fundamenski, Plasma Phys. Controlled Fusion 53, 095002 (2011)

${ }^{30}$ D. A. Mossessian, R. J. Groebner, R. A. Moyer, T. H. Osborne, J. W. Hughes, M. Greenwald, A. Hubbard, and T. L. Rhodes, Phys. Plasmas 10, 689 (2003).

${ }^{31}$ M. Groth, J. Boedo, N. Brooks, R. Isler, A. Leonard, G. Porter, J. Watkins, W. West, B. Bray, M. Fenstermacher, R. Groebner, R. Moyer, D. Rudakov, J. Yu, and L. Zeng, Nucl. Fusion 49, 115002 (2009).

${ }^{32}$ M. N. A. Beurskens, T. H. Osborne, L. D. Horton, L. Frassinetti, R. Groebner, A. Leonard, P. Lomas, I. Nunes, S. Saarelma, P. B. Snyder, I. Balboa, B. Bray, K. Crombe, J. Flanagan, C. Giroud, E. Giovannozzi, 
M. Kempenaars, N. Kohen, A. Loarte, J. Lonnroth, E. de la Luna, G. Maddison, C. Maggi, D. McDonald, G. McKee, R. Pasqualotto, G. Saibene, R. Sartori, E. R. Solano, W. Suttrop, E. Wolfrum, M. Walsh, Z. Yan, L. Zabeo, and D. Zarzoso, Plasma Phys. Controlled Fusion 51, 124051 (2009).

${ }^{33}$ S. Braginskii, Rev. Plasma Phys. 1, 205 (1965).

${ }^{34}$ J. R. Myra, D. A. D'Ippolito, X. Q. Xu, and R. H. Cohen, Phys. Plasmas 7, 4622 (2000).

${ }^{35}$ J. W. Connor, R. J. Hastie, H. R. Wilson, and R. L. Miller, Phys. Plasmas 5, 2687 (1998).

${ }^{36}$ D. Ryutov and R. Cohen, Contrib. Plasma Phys. 44, 168 (2004); in 9th International Workshop on Plasma Edge Theory in Fusion Devices, University of California, San Diego, CA, SEP 03-05, 2003.

${ }^{37}$ A. Zeiler, J. Drake, and B. Rogers, Phys. Plasmas 4, 2134 (1997).

${ }^{38}$ B. N. Rogers and W. Dorland, Phys. Plasmas 12, 062511 (2005).
${ }^{39}$ P. Ricci, B. N. Rogers, and S. Brunner, Phys. Rev. Lett. 100, 225002 (2008).

${ }^{40}$ J. T. M. Antonsen, J. F. Drake, P. N. Guzdar, A. B. Hassam, Y. T. Lau, C. S. Liu, and S. V. Novakovskii, Phys. Plasmas 3, 2221 (1996).

${ }^{41}$ J. F. Drake, Y. T. Lau, P. N. Guzdar, A. B. Hassam, S. V. Novakovski, B. Rogers, and A. Zeiler, Phys. Rev. Lett. 77, 494 (1996).

${ }^{42}$ D. Dobrott, D. B. Nelson, J. M. Greene, A. H. Glasser, M. S. Chance, and E. A. Frieman, Phys. Rev. Lett. 39, 943 (1977).

${ }^{43}$ T. M. Antonsen, Phys. Rev. Lett. 41, 33 (1978).

${ }^{44}$ P. N. Guzdar, L. Chen, P. K. Kaw, and C. Oberman, Phys. Rev. Lett. 40, 1566 (1978).

${ }^{45}$ S. Coda and TCV Team, Nucl. Fusion 51, 094017 (2011).

${ }^{46}$ E. Anderson, Z. Bai, C. Bischof, S. Blackford, J. Demmel, J. Dongarra, A. G. J. Du Croz, S. Hammarling, A. McKenney, and D. Sorensen, LAPACK User's Guide, 3rd ed. (SIAM, Philadelphia, 1999).

${ }^{47}$ N. J. Higham, SIAM J. Matrix Anal. Appl. 26, 1179 (2005). 